\title{
Submillimeter Polarimetry with PolKa, a reflection-type modulator for the APEX telescope
}

H. Wiesemeyer ${ }^{1}$, T. Hezareh ${ }^{1}$, E. Kreysa ${ }^{1}$, A. Weiss ${ }^{1}$, R. Güsten ${ }^{1}$, K.M. Menten ${ }^{1}$, G. Siringo ${ }^{2}$, F. Schuller ${ }^{2}$, A. Kovacs ${ }^{3,4}$

(1) Max-Planck-Institute for Radio Astronomy, Bonn, Germany

(2) European Southern Observatory, Santiago, Chile

(3) University of Minnesota, Minneapolis, MN 55405

(4) California Institute of Technology, Pasadena, CA 91125

hwiese@mpifr.de

Received —

For submission to PASP 


\begin{abstract}
Imaging polarimetry is an important tool for the study of cosmic magnetic fields. In our Galaxy, polarization levels of a few up to $\sim 10 \%$ are measured in the submillimeter dust emission from molecular clouds and in the synchrotron emission from supernova remnants. Only few techniques exist to image the distribution of polarization angles, as a means of tracing the plane-of-sky projection of the magnetic field orientation. At submillimeter wavelengths, polarization is either measured as the differential total power of polarization-sensitive bolometer elements, or by modulating the polarization of the signal. Bolometer arrays such as LABOCA at the APEX telescope are used to observe the continuum emission from fields as large as $\sim 0.2$ in diameter. Here we present PolKa, a polarimeter for LABOCA with a reflection-type waveplate of at least $90 \%$ efficiency. The modulation efficiency depends mainly on the sampling and on the angular velocity of the waveplate. For the data analysis the concept of generalized synchronous demodulation is introduced. The instrumental polarization towards a point source is at the level of $\sim 0.1 \%$, increasing to a few percent at the $-10 \mathrm{db}$ contour of the main beam. A method to correct for its effect in observations of extended sources is presented. Our map of the polarized synchrotron emission from the Crab nebula is in agreement with structures observed at radio and optical wavelengths. The linear polarization measured in OMC1 agrees with results from previous studies, while the high sensitivity of LABOCA enables us to also map the polarized emission of the Orion Bar, a prototypical photon-dominated region.
\end{abstract}

Subject headings: Astronomical techniques and data analysis: polarimetry 


\section{Introduction}

Magnetic fields are important constituents in the interstellar medium (ISM) and are theoretically shown to control many physical processes including formation and fragmentation of molecular clouds (e.g., Heitsch et al. 2005; Hennebelle et al. 2008), and regulation of the process of star formation (e.g., Mouschovias \& Ciolek 1999). Observational studies, on the other hand, are essential to provide constraints on the existing numerical models. However, magnetic field observations require state-of-the art instruments and intricate observing and data reduction techniques.

Submillimeter polarimetry is one of the best tools for characterizing interstellar magnetic fields, because in most environments commonly observed aspherical dust grains are aligned with their principle axis of major inertia along the magnetic field lines. The physics of grain alignment is extremely rich and complex; Lazarian (2007) and (with

emphasis on cold clouds) Draine (2004) review the underlying theory. For decades dissipation of rotational energy in paramagnetic grains (Davis \& Greenstein 1951), set into suprathermal rotation by various torques (Purcell 1979), was considered the dominant process. Towards the end of the 90 s it became clear that anisotropic radiative torques do not only spin up the grains (Draine \& Weingartner 1996), but also align them efficiently with respect to the magnetic field (Draine \& Weingartner 1997). This alignment mechanism works for larger grains with effective radii $a_{\text {eff }} \gtrsim 0.1 \mu \mathrm{m}$, while smaller grains are at most weakly aligned (Kim \& Martin 1995; Draine \& Fraisse 2009). More recent work on radiative torques is reviewed by Lazarian \& Hoang (2011) with further references therein. We also note that dust grains exposed to sub- or super-sonic gas flows are expected to be mechanically aligned with their long axis perpendicularly to the magnetic field (Lazarian \& Hoang 2007). In summary, for molecular clouds the thermal emission of dust is linearly polarized orthogonally to the field lines, and a polarization map will reveal the direction 
of the plane-of-sky component of the magnetic field. For equipartition between magnetic pressure and turbulent and thermal presure, its strength can be determined by means of the Chandrasekhar-Fermi method (Chandrasekhar \& Fermi 1953). This technique was originally applied to estimate the magnetic field strength in Galactic spiral arms. Chandrasekhar and Fermi used the dispersion of polarization vectors with respect to the large-scale magnetic field, known, from the dichroic absorption of starlight (Hiltner 1951), to be generally aligned with the Galactic plane. Hildebrand et al. (2009) and Houde et al. (2009) have developed the CF-method further, accounting for the distortion of the magnetic field by turbulence; their analysis does not need to assume any model for the large-scale field. MHD simulations have shown (Heitsch et al. 2001) that modified CF-methods are astonishingly robust, and that order-of-magnitude estimates are possible even in regions that are not dominated by hydromagnetic turbulence.

This work deals exclusively with linear polarization. While in the NIR a substantial fractional circular polarization can be expected for light scattered by aligned dust grains ( $\simeq 15 \%$ have been observed in the BN object in OMC1, Matsumura \& Bastien 2009) or in the $\lambda 3.1 \mu \mathrm{m}$ feature of water ice (e.g., Aitken et al. 2006 for a summary of detections and further references), at sub-millimeter wavelengths we expect at most a weak signal. - In practice, the polarimetry of dust emission is completed by analyzing the circular and linear polarization of suitable spectral lines (Zeeman and Goldreich-Kylafis effects, for a review see e.g., Crutcher 2012).

Sub-millimeter imaging polarimetry is not limited to dust polarization, but also useful to study the synchrotron emission from e.g., plerion-type supernova remnants. While polarimetry reveals the magnetic field structure, observations into the far-infrared allow to determine the break in the spectral energy distribution and therefore the strength of the magnetic field (Marsden et al. 1984, Salter et al. 1989), and to trace the production of dust 
by the progenitor star (Green et al. 2004).

As the theory and analytical methods evolved, technological progress has been made in submillimeter imaging and polarimetry. Since low-mass protostars in nearby $(d \sim 100 \mathrm{pc})$ star forming regions and protoclusters in regions where massive stars form ( $d \sim 1 \mathrm{kpc}$ ) extend over scales corresponding to the limited field-of-view of (sub)millimeter interferometers (at most a few minutes of arc), remarkable results have been achieved thanks to their dual-polarization capabilities and powerful digital cross-correlators (e.g., Girart et al. 2013). On larger scales, inaccessible to interferometers, the imaging surveys done with the Herschel space observatory (e.g., a survey of the Gould Belt in which low-mass stars form, André et al. 2010, and the HOBYS survey for regions forming massive stars, Motte et al. 2010), revealed filamentary structures across large areas on the sky. While the formation of these filaments out of the warm, neutral phase of the interstellar medium has motivated the aforementioned and other theoretical studies (e.g., Heitsch et al. 2005; Hennebelle et al. 2008), the large-scale magnetic field and therefore its dynamical role are still poorly constrained by observations. In the past decades, several wide-field bolometer cameras at various submillimeter observatories have therefore been equipped with polarimeters. Table 1 summarizes their main features along with published first-light observations. Submillimeter interferometers are not considered because they are blind to the large spatial scales addressed by this paper. Likewise, spectro-polarimeters are omitted because they are designed for different, yet often complementary studies (e.g., correlation polarimeters such as XPOL, Thum et al. 2008).

For incoherent detectors, such as in bolometer arrays, two methods to separate polarized from unpolarized radiation are possible, namely (1) differential total power measurements, and (2) modulations of the plane of polarization. In the first technique, the total power of detectors that are sensitive to polarizations at position angles $0^{\circ}, \pm 45^{\circ}$ 
and $90^{\circ}$ yields Stokes parameters $I, Q$ and $U$. This method is used in the low- and high frequency instruments aboard the Planck satellite (Leahy et al. 2010, Rosset et al. 2010), employing polarization-sensitive elements (Kuo et al. 2008), and for the balloon-borne BLAST-Pol experiment (Fissel et al. 2010). In the second method the plane of polarization is rotated periodically or stepwise, allowing to measure the polarization of the incident radiation field with polarization-sensitive detectors, or, by means of a polarization analyzer (e.g., a grid), also with detectors otherwise insensitive to polarization. The modulator can be either a classical waveplate operating in transmission or a tunable, reflecting polarizer (see Table 1).

In both methods the measurements of the Stokes parameters received from a given position in the sky are not strictly simultaneous. A too slow detection cycle is inadequate especially for ground-based observatories, plagued by atmospheric instabilities. In the first method, the resulting $1 /$ f noise can be suppressed by scanning fast enough. In the second method, the rotation of the plane of polarization needs to be faster than the atmospheric total power fluctuation, suggesting a continuous rather than stepwise rotation. The suppression of the 1/f noise in Stokes I, measured simultaneously with Stokes Q and U, still requires fast scanning. We will come back to this topic in section 3 .

This work describes PolKa (Siringo et al. 2012, 2004), the polarimeter for the Atacama Pathfinder Experiment (APEX, Güsten et al. 2006). PolKa, as a fast reflection-type polarization modulator, belongs to the second type of polarimeters. It is used with LABOCA, the Large APEX Bolometer Camera (Siringo et al. 2009). LABOCA operates at $\lambda 870 \mu \mathrm{m}$ and consists of 295 semiconductor bolometers, detecting the temperature rise due to the absorption of radiation. The bolometers are arranged in an hexagonal grid with two beam spacing, providing an instantaneous field-of-view of 0.2 . For the PolKa observations, 230 pixels offer an acceptable noise level. 
The plan of the paper is as follows: Section 2 describes the hardware of the rotating half-waveplate, its working principle, the measurement equation and the characterization and calibration of the device. Section 3 provides a detailed description of the data reduction methods such as correlated-noise and bandpass filtering. We also introduce novel techniques to demodulate the signals by a generalized synchronous demodulation and to remove instrumental artifacts. These techniques are indispensable for imaging polarimetry and were specifically created for PolKa. Section 4 presents the first observational results from PolKa with a reliable polarization angle calibration. We conclude the paper with section 5 and refer the reader interested in the relevant mathematical methods to the appendix.

\section{Design of reflection-type polarization modulators}

Polarization transforming reflectors were devised already more than two decades ago (Howard et al. 1986; Prigent et al. 1988), but in (sub-)millimeter astronomy they have been used only twice. Several designations for devices of this kind exist in literature, for the purpose of this paper we refer to them as reflecting polarization modulator (hereafter RPM). Shinnaga et al. (1999) and Siringo et al. (2004) describe, respectively, the systems installed at the NRO 45m (for spectral line polarimetry) and the SMTO 10m (with a 19-channel bolometer array operating at $\lambda 870 \mu \mathrm{m})$ telescopes. Modulators of this kind have the double advantage that their reflectivity is excellent (closer to $100 \%$ than the transmission of classical waveplates) and that they can be tuned within a wide wavelength range (only limited by the design of the grids). 


\subsection{Working principle}

RPMs are simple yet efficient optical equivalents to birefringent materials (Fig. 1): In a parallel assembly of a grid and a mirror, mounted at an adjustable distance, the component

of the incident radiation that is polarized parallel to the wires can excite an electromagnetic mode and is reflected off, while the component polarized perpendicular to the wires is transmitted and then reflected into the outgoing ray where it is superposed to the other ray, with a delay-induced phase difference. In a birefringent material, these two components correspond to the "fast" and the "slow" rays, respectively. If the device is tuned such that the phase difference between the two rays is $180^{\circ}$, the overall effect is that of a classical half-waveplate, i.e., transmitting both polarized and unpolarized radiation, but rotating the plane of polarization by the double position angle of the grid. When a linearly polarized incident signal is measured with a polarization-sensitive bolometer element (or through an analyzer grid, for elements insensitive to polarization), a rotation of the waveplate therefore modulates the received power. The amplitude and the phase of the modulation (which for a constant rotation speed is sinusoidal) then yields the linear polarization and its polarization angle, respectively.

\subsection{Description of the hardware}

The Cassegrain focal plane of the APEX telescope is reimaged to the bolometer array of LABOCA (Siringo et al. 2009) with three concave mirrors, two flat mirrors and a lens. The aperture ratio of the Cassegrain focus, $F / D=8.0$, is reduced to $F / D=1.75$ in the focus of LABOCA. We stress that the accomodation of a polarimeter was in the design specifications of the tertiary optics right from the beginning. In 2009, when PolKa was permanently installed, it simply replaced one of the two flat mirrors and a filterwheel with two analyzer grids was added. The modulator of PolKa (Fig. 2) was designed and 
manufactured by the Fraunhofer Institute for Applied Optics and Precision Engineering (Fraunhofer IOF, Jena, Germany). The grid for the modulator was made in the division for submillimeter technologies at MPIfR, has an aperture of $246 \mathrm{~mm}$ and is made of $20 \mu \mathrm{m}$ thick tungsten wires (Siringo et al. 2012). For the analyzer, two identical grids were contributed by RWTH University Aachen (Germany), each of $146 \mathrm{~mm}$ aperture and made of gold-coated tungsten wires. The two analyzer grids are mounted at different position angles in order to suppress systematic effects, an equipment feature to which we will come back in section 2.6. As shown in Fig. 2 (right panel), the analyzer grid is used only in transmission. The smooth rotation of the grid-mirror unit is ensured by an air bearing. It consists of a rotor (pale orange in Fig. 2) and a stator (shown in orange). The compressed air enters through a channel machined into the stator and flows from the center of the bearing to the outside. The surface of the bearing is made of two hemispheres. This ensures that the bearing works in any orientation, which for a Cassegrain cabin is evidently an indispensable feature. We operate the air bearing at a pressure of 4 bar, which is appropriate given the altitude of the observatory $(5105 \mathrm{~m})$. The position angles are read by an encoder; in practice, a time stamp is written for each crossing of a well defined reference position. Interpolations between two successive reference crossings then provide the position angles for each record in the bolometer data stream, sampled at a speed of $1 \mathrm{kHz}$. The whole dataset (bolometer total power counts, and the speed and position angle of the waveplate) are then downsampled to a frequency in the range from 25 to $50 \mathrm{~Hz}$ and written to raw data files (in multi-beam FITS format, Muders et al. 2006), which are subsequently analyzed by the data processing software described in section 3 . 


\subsection{Measurement equation}

The measurement equation accounts for the multiple reflections by defining a suitable coordinate frame for the description of linear polarizations and therefore Stokes $Q$ and $U$. The rotations and inversions of the coordinate system in the path of the beam are then described by successive similarity transformations of the coherency matrix (Born \& Wolf 1999). This algebraic formulation of polarimetry owes its power to the capacity to represent both mixed polarization states (like Müller matrices) and phase information (like complex Jones vectors). For details, we refer to appendix A. Here we present the result of the calculation, which is provided in full length in appendix B. We define the coordinate system in the focal plane, located between the vertex of the telescope and its tertiary mirror, as shown in Fig. 3. In the following, we will refer to this frame as "Cassegrain coordinate system" with Stokes parameters $Q_{\mathrm{c}}, U_{\mathrm{c}}$ defined such that the polarization angle

$$
\psi_{\mathrm{c}}=\frac{1}{2} \operatorname{atan} 2\left(U_{\mathrm{c}}, Q_{\mathrm{c}}\right)=\frac{1}{2} \operatorname{Arg}\left(U_{\mathrm{c}}+i Q_{\mathrm{c}}\right)
$$

is measured counter-clockwise from the positive $\mathrm{x}$-axis (IEEE definition), as viewed from the tertiary to the secondary mirror. The resulting measurement equation reads

$$
\begin{aligned}
S=\frac{1}{2}\{ & I-\frac{V}{2} \sin (2 \varphi-\Delta \theta) \sin \Phi \\
& \left.+Q_{\mathrm{c}}\left[\cos ^{2}(2 \varphi-\Delta \theta)+\sin ^{2}(2 \varphi-\Delta \theta) \cos \Phi\right]-\frac{U_{\mathrm{c}}}{2} \sin (4 \varphi-2 \Delta \theta)(1-\cos \Phi)\right\}
\end{aligned}
$$

with the Stokes parameters $I, Q_{\mathrm{c}}, U_{\mathrm{c}}$ and $V$. The signs of the terms for Stokes $V$ and $U$ are opposite to the sign of the Stokes $Q$ term because PolKa is a reflecting polarimeter. $\varphi$ is the position angle of the grid but projected onto a plane normal to the incoming ray, measured counter-clockwise from the plane of incidence. It is related to the position angle $\varphi_{0}$ of the wires of the grid (as read out by the encoder) by $\varphi=\arctan \left(\cos \alpha \tan \varphi_{0}\right)$, where $\alpha$ is the angle of incidence defined in Fig. 1. Because of the relatively small angle of incidence in our setup $(\alpha=16.23)$, the difference between $\varphi$ and $\varphi_{0}$ is at most 1.17 , so 
that the sampling of position angles is not too distorted and remains reasonably regular. $\Phi$ is the phase difference created by the delay between the reflected and transmitted (thus, orthogonal) polarization. The angle $\Delta \theta$ accounts for the rotation of the $(x, y)$ plane in Fig. 3 that occurs when the beam is downfolded, and for the orientation of the analyzer grid. More precisely, the rotation angle $\Delta \theta$ is given by

$$
\Delta \theta=\theta_{\mathrm{PA}}-\theta_{\mathrm{FP}}
$$

where $\theta_{\mathrm{PA}}$ is the rotation of the beam occurring between the RPM and the analyzer grid (measured with respect to its wires). $\theta_{\mathrm{FP}}$ describes the beam rotation between the Cassegrain focal plane and PolKa, measured counter-clockwise with respect to the $\mathrm{x}$-axis in the focal plane (Fig. 3).

We deliberately show the measurement equation (2) in full generality because it allows to estimate the precision needed to tune the RPM, and to evaluate the bandwidth smearing that will be addressed below. We also keep Stokes $V$ in equation (2), although in view of the aforesaid it can be expected to be small in most applications. Incidentally, it may still be produced by the spurious conversion of Stokes $I$ into Stokes $V$ arising when a grid is mounted in a divergent beam with its wires parallel to the plane of incidence $\mathrm{Chu}$ et al. 1975, Thum et al. 2008). Obviously, this occurs in every other modulation cycle. However, even if this instrumental conversion was a first-order effect, its impact on the measured modulation would be of second order, owing to the half-waveplate tuning and to the relatively small phase error introduced by an imperfect tuning and/or the bandwidth effect discussed below.

We finally note here that a fast polarization modulation may also be generated by periodically varying the delay-induced phase difference $\Phi$. As shown by Chuss et al. (2006) and Krejny et al. (2008), a sinusoidal modulation can then be achieved by adding a second polarization transforming reflector, rotated by $-45^{\circ}$ with respect to the first one 
(cf. Table 1). In such a design, known as a dual variable-delay polarization modulator (dual VPM), the system can be kept free from unwanted oscillations by using piezo-electric actuators (the translational polarization rotator introduced by Chuss et al. $2012 \mathrm{~b}$ is a further development). It seems fair to say that in both approaches the stability requirement makes the system more complex, either by adding a second VPM, or, as in our case, an air-bearing. A way to enable an even faster modulation without moving parts is the Faraday rotation modulator (Keating 2009) using a ferrite dielectric waveguide. This kind of magneto-optical devices has a promising performance in the polarimetry of the cosmic microwave background and of diffuse, large-scale Galactic dust emission at frequencies up to $150 \mathrm{GHz}$ (Moyerman et al. 2013). However, to implement this technology for large detector arrays at higher frequencies is very challenging.

We now rewrite the measurement equation (2) for a vanishing Stokes $V$, but introduce efficiency factors $\eta_{\mathrm{tm}}, \eta_{\mathrm{bp}}$ and $\eta_{\mathrm{ts}}$ describing the optical transmission, and the bandpassand time-smearing, respectively:

$$
S=\frac{1}{2} \eta_{\mathrm{tm}}\left\{I+\eta_{\mathrm{bp}} \eta_{\mathrm{ts}}\left[Q_{\mathrm{c}} \cos (4 \varphi-2 \Delta \theta)-U_{\mathrm{c}} \sin (4 \varphi-2 \Delta \theta)\right]\right\}
$$

The efficiency factors will be quantified in the next section. A rotation of the analyzer grid by $90^{\circ}$ obviously inverts the signs of $Q_{\mathrm{c}}$ and $U_{\mathrm{c}}$. By adding the signals from measurements with orthogonal analyzer orientations it is in principle possible to separate Stokes $I$ from Stokes $Q$ and $U$. This requires a strict synchronization between the slew motion of the telescope and the waveplate rotation when observing in on-the-fly mode. In practice such a synchronization is difficult to achieve and its failure leads to a substantial loss of usable data. At best, such an approach may work for fast on-off observations of compact sources, an application not considered in this paper. For the extended sources we focus on here, scanning the sky in the on-the-fly mode and thereby obtaining a Nyquist-critical sampling, is the observing method of choice for which a fault-tolerant system (yet accurately 
tracing actual values) proves to be more efficient. We will show in section 3 that the Stokes parameters can be separated by a dedicated time series analysis. Notwithstanding, using two analyzer grids at different orientations may suppress a part of the instrumental polarization, which we will characterize below.

In order to obtain, for a given point of the mapped area, a stationary Stokes $Q$ and $U$, we transform $\left(Q_{\mathrm{c}}, U_{\mathrm{c}}\right)$ to the equatorial reference frame. From the transformation of the left-handed Cassegrain coordinates $(x, y)$ to offsets $(\Delta \alpha \cos \delta, \Delta \delta)$ in the right-handed system of the tangential plane,

$$
\left(\begin{array}{c}
\Delta \alpha \cos \delta \\
\Delta \delta
\end{array}\right)=\left(\begin{array}{rr}
\cos \eta & -\sin \eta \\
-\sin \eta & -\cos \eta
\end{array}\right)\left(\begin{array}{c}
\Delta x \\
\Delta y
\end{array}\right)
$$

with $\eta$ the parallactic angle, we can readily derive the corresponding transformation of Stokes $\left(Q_{\mathrm{c}}, U_{\mathrm{c}}\right)$ to $\left(Q_{\mathrm{eq}}, U_{\mathrm{eq}}\right)$, leading in equations (2) and (4) to the substitution $Q_{\mathrm{c}} \rightarrow Q_{\mathrm{eq}}, U_{\mathrm{c}} \rightarrow-U_{\mathrm{eq}}, \Delta \theta \rightarrow \Delta \theta+\eta$. In the IAU definition, which differs from the IEEE definition, the polarization angle is measured counter-clockwise from north and is now given by

$$
\tau_{\mathrm{IAU}}=\frac{1}{2}\left[\pi-\operatorname{atan} 2\left(U_{\mathrm{eq}}, Q_{\mathrm{eq}}\right)\right]
$$

It should be kept in mind that $Q_{\mathrm{c}}$ and $U_{\mathrm{c}}$ consist of an intrinsic signal and a polarization of instrumental origin, arising mainly in the tertiary optics.

The fractional linear polarization $p_{\mathrm{L}}$ used throughout the rest of this paper is given by

$$
p_{\mathrm{L}}=\frac{\sqrt{Q_{\mathrm{eq}}^{2}+U_{\mathrm{eq}}^{2}}}{I} .
$$

Stokes $Q_{\text {eq }}$ and $U_{\text {eq }}$ are fraught with systematic and random errors. The former will be removed in the correction for instrumental polarization (see section 2.6), but random errors still lead to a bias and therefore an overestimate of $p_{\mathrm{L}}$ (see e.g., Wardle \& Kronberg 1974). However, it can be shown, both with a Monte-Carlo simulation and analytically, that if the 
most likely values of $Q_{\text {eq }}$ and $U_{\text {eq }}$ are used (in general median values), no such corrections need to be done (for details see Section 3). Therefore, $p_{\mathrm{L}}$ and $\tau_{\mathrm{IAU}}$ are calculated only at the last data reduction stage.

\subsection{Transmission properties and flux calibration}

The modulation efficiency can be decomposed into the three factors $\eta_{\mathrm{bp}}, \eta_{\mathrm{ts}}$ and $\eta_{\mathrm{tm}}$. From equation (4) one can see that only the third factor affects all Stokes parameters, while the other two factors only affect Stokes parameters $Q_{\mathrm{c}}$ and $U_{\mathrm{c}}$.

The first factor, $\eta_{\mathrm{bp}}$ accounts for the fact that an optimal tuning can only be achieved at a nominal frequency, while the bandpass of LABOCA, defined by a set of cold filters at the liquid nitrogen and helium-4 stages, extends over $60 \mathrm{GHz}$ (Fig. 5 in Siringo et al. 2009). Even if the grid and mirrors had a 100\% efficiency, the modulation would suffer from a

loss because the optimal half-wave phase shift $(\Phi=\pi)$ can only be achieved at a fixed frequency. Three micrometer screws allow to fine-tune the distance between the mirror and the grid, $d(\mathrm{M}, \mathrm{G})$. At the passband-weighted center frequency of $344 \mathrm{GHz}\left(\lambda_{0}=870 \mu \mathrm{m}\right)$ and for our angle of incidence, $\alpha=16.23$,

$$
d(\mathrm{M}, \mathrm{G})=\frac{\Phi \lambda_{0}}{4 \pi \cos \alpha}=\frac{\lambda_{0}}{4 \cos \alpha}=227 \mu \mathrm{m} .
$$

The tuning distance is $217 \mu \mathrm{m}$ to account for the finite thickness of the tungsten wires. The zero position of the micrometer screws has been confirmed by the disappearance of the Moiré pattern arising for a finite mirror-grid distance. At a wavelength offset $\Delta \lambda$ from $\lambda_{0}$, the phase is shifted from its optimum value $\Phi=\pi$ to

$$
\Phi=\pi+\Delta \Phi=\pi\left(1-\frac{\Delta \lambda}{\lambda_{0}+\Delta \lambda}\right) .
$$

Equation (2) is then used to calculate the modulation across the spectral bandpass. The latter may be modified by the atmospheric transmission, but for acceptable observing 
conditions, the loss of modulation efficiency does not strongly depend on the weather. For a water vapor column of $0.7 \mathrm{~mm}, 50^{\circ}$ elevation, and $553 \mathrm{hPa}$ ambient pressure the resulting modulation efficiency is $\eta_{\mathrm{bp}}=99.3 \%$. Because $\Delta \Phi<0.1 \mathrm{rad}$, the bandpass smearing is a second order effect which explains why it affects the polarization efficiency only weakly.

The second factor, $\eta_{\mathrm{ts}}$, results from the time smearing, i.e., the dilution of the modulation due to the elementary integration time step $\Delta t$, given by the sinc function

$$
\eta_{\mathrm{ts}}=\sin (2 \omega \Delta t) /(2 \omega \Delta t)
$$

where $\omega=2 \pi f_{0}$ is the angular speed of the waveplate rotation. In practice, we use $f_{0}=1.56 \mathrm{~Hz}$ and for integration intervals of $\Delta t=20$ or $40 \mathrm{msec}$ the dilution factors are 97 and $90 \%$, respectively.

The third factor, $\eta_{\mathrm{tm}}$, can be calculated numerically from the optical properties of the polarizer (i.e., the grid labeled $G$ in Fig. 1), namely its reflectance, $R_{\|}$and transmission, $T_{\perp}$ for the radiation power polarized parallel, respectively perpendicular, to the wire grids (Chuss et al. 2012a, further references therein). In the limiting case where $\lambda>2 p, \lambda \gg a$, and $a / p<1 / 2 \pi$ (where $p$ is the spacing between successive wires and $a$ their diameter) one can calculate $R_{\|}$and $T_{\perp}$ from (Lamb 1898)

$$
R_{\|}=\frac{1}{1+\left(\frac{2 p \cos \alpha}{\lambda_{0}} \ln \frac{p}{\pi a}\right)^{2}}, T_{\perp}=1-R_{\perp}=\frac{1}{1+\left(\frac{\pi^{2} a^{2}}{2 \lambda_{0} p}\right)^{2}} .
$$

For PolKa, $p=(63 \pm 18) \mu \mathrm{m}$ (the error has been determined from measurements under a microscope) and $a=20 \mu \mathrm{m}$. Therefore, the filling factor $a / p$ does not obey the last of the three conditions above. Chambers et al. (1980) used a Green function method to semi-analytically derive the transmission of wire grids. Using their results Chambers et al. 1988) for our filling factor of $a / p=0.317 \pm 0.091$ and the spacing of $p / \lambda=0.072 \pm 0.021 \mu \mathrm{m}$, we find $R_{\perp}=0.1 \%(+0.4,-0.1) \%$ for normal incidence, for our case of a slightly oblique incidence the resulting $R_{\perp}$ would decrease even more. We note (1) that the application of 
the approximative Eq. 11 yields a value for $R_{\perp}$ well within the errors due to the measured variance of $p$, and (2) that the impact of the latter is small due to the low ratio of $p / \lambda$ (cf. Fig. 13 of Chambers et al. 1986). Khazan (2002) showed with terahertz time domain spectroscopy that the theoretical predictions from Green's function describe the actual measurements of Tungsten wire grids similar to ours fairly well. This discussion also strongly suggests that the polarization efficiency $\eta_{\mathrm{bp}} \eta_{\mathrm{ts}}$ in Eq. 4 is dominated by the time smearing factor $\eta_{\mathrm{ts}}$.

We are therefore confident that $\eta_{\mathrm{tm}}$ is close to 0.99 (the waveplate mirror is of optical quality and therefore leads to no loss of efficiency). Its measurement by means of primary calibrators is notoriously difficult. When PolKa started its operation in December 2011 the flatfielding was done on Mars without the modulator and analyzer grids. Folding the fluxes predicted by the model of Rudy et al. (1987) (and calculated with the online tool provided by Butler 2008) into the bandpass of LABOCA yields a calibration factor of 4.74 Jy/ $\mu \mathrm{V}$. The model by Lellouch \& Amri (2006) yields marginally ( 1\% at $300 \mathrm{GHz})$ higher continuum fluxes. After insertion of the modulator grid calibration maps on Mars, taken without the analyzer grid, showed no significant flux loss: During the two-week campaign, we measured daily the flux rise of Mars (the planet approached its March 2012 opposition). The uncertainty of the relative flux scale is typically $2 \%$. Comparing the model with the measured fluxes yields a flux calibration factor of $4.63 \mathrm{Jy} / \mu \mathrm{V}$. The accuracy is limited by the uncertainty of the model flux, estimated to $\sim 5 \%$. - 19 Uranus maps, made to characterize the instrumental polarization (see section 2.6), yield a calibration factor of 4.75 Jy/ $/ \mu \mathrm{V}$, using the Uranus model ESA-4 Orton et al. 2014, estimated to be accurate to $2-3 \%$ ) and assuming that the analyzer grid has a transmission of $100 \%$ in one polarization and no cross polarization. The calibration factors agree within $5 \%$ with respect to the mean value, $4.7 \mathrm{Jy} / \mu \mathrm{V}$. We stress that the observations of Uranus, unlike those of Mars, were done in polarimetry mode, which involves a more intricate data reduction (section 3), while 
the flux scale is preserved. The good agreement between the calibrations done without grids, with the modulator grid, and then with the analyzer grids sets a lower limit of $95 \%$ to the transmission $\eta_{t m}$ of PolKa.

Our calibration factor falls $27 \%$ below the value determined by Siringo et al. (2009). One reason for this discrepancy is the determination of the opacity correction. We obtain it from the precipitable water vapor $(p w v)$ measured once per minute with the APEX $183 \mathrm{GHz}$ radiometer and converted to a bandpass-weighted zenith opacity. A consistent and reproducible calibration is also important in view of the processing of polarization data obtained under varying weather conditions (see section 3). We use the am transmission mode $\rfloor^{1}$ predicting a zenith opacity of $\tau_{\nu}=b_{\nu}\left(p_{\mathrm{amb}}\right) p w v+c_{\nu}\left(p_{\mathrm{amb}}\right)$, where the coefficients $b_{\nu}$ and $c_{\nu}$ parameterize the "wet" and "dry" atmosphere and depend on frequency and outside pressure (Guan et al. 2012).

An accurate comparison of our calibration with that of other polarimeters is difficult. Different bolometer arrays have different spectral bandpasses, therefore flux measurements in sources with strong spectral indices and substantial contributions from spectral lines vary from instrument to instrument, e.g. for OMC1 (section 4.3) where spectral lines in the SCUBA bandpass contribute up to 50\% to the observed flux (Groesbeck 1995; Johnstone \& Bally 1999). For Tau A (the Crab nebula, section 4.2), whose flux is dominated by synchrotron emission with a relatively flat spectral index $\left(S \propto \nu^{-0.3}\right)$, our peak flux, applying the calibration factors derived from Mars and Uranus, differs, after correcting for the slightly different beam sizes, by only 3.6\% from that of Green et al. (2004), measured with SCUBA at $\lambda 850 \mu \mathrm{m}$.

\footnotetext{
${ }^{1}$ Scott Paine, SMA technical memo \#152, version 7.2, February 2012
} 


\subsection{Polarization angle calibration}

As stated in section 1, the most important quantity for the analysis of magnetic fields by means of polarimetry of dust or synchrotron emission is the polarization angle. Its accuracy depends on the instrumental conversion between Stokes $I$ on the one hand and Stokes $Q$ and $U$ on the other hand, and between Stokes $Q$ and $U$. The first conversion can be corrected by means of an unpolarized calibrator (we used Uranus, see section 2.6), while the second one arises in the tertiary optics and can be measured with an additional polarizer. In March 2013 we performed a series of calibrations with a high-quality grid mounted in the focal plane of the telescope (Fig. 3), in reflection for vertical polarization as defined in the Cassegrain reference frame (i.e., with the wires along the y-axis of the Casssegrain coordinate system, perpendicular to the elevation axis of the telescope). The grid was mounted to better than $1^{\circ}$ accuracy, and we can safely assume a fully polarized signal with $Q_{\mathrm{c}} / I=-1$ and $U_{\mathrm{c}} / I=0$. We obtained $\Delta \theta=114.6$ and 2.4 for the two analyzer grids mounted on the filterwheel and therefore an absolute polarization angle calibration with an accuracy well below the limitation by the sensitivity. The difference between these observed angles is confirmed by a direct measurement to within 0.2 . Our absolute polarization angle calibration was further confirmed by observations of celestial sources that will be presented below together with other first light observations. The statistical error of the polarization angle is given, for equal noise contributions from Stokes $Q$ and $U$ (which is the case here), by $\sigma_{\psi}=\sigma_{\mathrm{pL}} / 2 p_{\mathrm{L}}$. In astronomical polarimetry, cutoffs of 2 to $3 \sigma_{\mathrm{pL}}$ are commonly used, i.e., statistical polarization angle errors of up to 14.3 respectively 9.5 are tolerated. 


\subsection{Instrumental polarization}

Thanks to its axisymmetric design, the level of instrumental polarization (hereafter we use the acronym IP) in a Cassegrain telescope is insignificant. As a matter of fact, the main contributions to IP arise from the tertiary optics in the receiver cabin (see also Thum et al. 2008 and further references therein), and ignoring them may lead to a severe misinterpretation of polarization data. In order to quantify and correct the IP, one ideally observes an unpolarized, unresolved source, e.g., a gas planet like Uranus. In principle, Mars and Mercury may also be useful. Their weak, radial polarization pattern cancels out if their disks remain unresolved (Mercury should be observed near full phase).

Fig. 4 shows the IP measured in on-the-fly mode on Uranus in the Cassegrain coordinate system. As in the map-making of Stokes I, all available LABOCA pixels have been used, scanning the planet at different times. The resulting polarization is therefore a weighted average of the IP at different distances from the optical axis. We expect sign changes of the instrumental Stokes Q and U across the field-of-view; therefore the IP in the averaged data partially cancels out. Because the sensitivity of the IP map is insufficient to be used for individual bolometer pixels, we cannot quantify to which extent this happens, but it seems fair to conclude that the IP in the final map, produced from the full dataset, is lower than for the individual pixels. The fractional linear IP towards the brightness peak of the Stokes $I$ beam amounts to $p_{\mathrm{L}}=(0.10 \pm 0.04) \%$. The spatial average within the $10 \mathrm{~dB}$ contour yields $(0.33 \pm 0.09) \%$. Using only the first analyzer grid yields $(0.17 \pm 0.06) \%$ at the peak position and $(0.47 \pm 0.18) \%$ within the $-10 \mathrm{~dB}$ contour, and with the second analyzer we obtained $(0.09 \pm 0.04) \%$ respectively $(0.37 \pm 0.36) \%$. These numbers demonstrate that the instrumental Stokes $Q$ and $U$ beams are wider than the Stokes $I$ beam, leading to a larger IP at off-source positions. They also show that using an analyzer grid at different orientations can help to decrease the IP level. A further reduction occurs because the 
IP pattern, whose polarization vectors are fixed in the Cassegrain coordinate system, is smeared out on the sky thanks to the parallactic rotation. However, the above numbers substantiate that the removal of IP with a dedicated correction algorithm is essential. In appendix C we present such a procedure that accounts for the detailed coupling of instrumental Stokes $Q$ and $U$ beam patterns to the brightness distribution on the sky. This approach is far more sophisticated than the a posteriori application of a constant fractional IP to the final polarization maps. An IP map like that shown in Fig. 4 can be used for our correction method, provided that in the sky plane the sampling of the source resembles as closely as possible to that of the IP calibrator. Nothing can be said about the IP below the $-10 \mathrm{~dB}$ contour due to the limitation of the dynamic range. To what extent this residual IP affects the accuracy of the measured polarization is difficult to say without deeper observations, each at several parallactic angles. It seems fair to say that only sensitive observations of weak polarizations $(\lesssim 10 \mathrm{mJy})$ are concerned when a strong source (Stokes $I \sim 100 \mathrm{Jy}$ ) is located in the error beam.

\section{Data reduction}

Thanks to the large field of view of LABOCA, PolKa is a polarimeter of choice for imaging the magnetic field structure of extended objects. So far fields as large as $10^{\prime}$ have been observed; larger areas require mosaics. The observing methods are the same as for non-polarimetric maps, i.e., a Nyquist-critical sampling is achieved by slew motions along spiral or linear patterns (see Siringo et al. 2009, section 8). In this mode, the noise-equivalent flux density per pixel is $55 \mathrm{mJy} \sqrt{\mathrm{s}}$ (sensitivity weighted mean value of all usable pixels after skynoise filtering, Siringo et al. 2009). However, owing to the modulation of the signal, the data reduction methods are more involved. PolKa is usually operated at a spinning frequency of $f_{0}=1.56 \mathrm{~Hz}$, or a modulation frequency of $f_{\mathrm{M}}=4 f_{0}=6.24 \mathrm{~Hz}$, so as 
to obtain four data records of 40 msec per modulation cycle. This frequency also allows to separate the modulation of the polarized flux from atmospheric fluctuations which remain below $\lesssim 3 \mathrm{~Hz}$, as demonstrated by the spectral power density (i.e., the Fourier transform of the autocorrelation function of the time series) shown in Fig. 5 (the apparent harmonic signal will be discussed in section 3.2.

A typical time series of PolKa data is shown in Fig. 6 and demonstrates the data reduction steps that will be discussed in the following. The signal can be decomposed into three contributions, namely into (1) a periodic, deterministic signal of instrumental origin, (2) a piecewise periodic, deterministic signal (the polarization received from the observed

source), and (3) a non-deterministic signal (the 1/f noise from the atmosphere, the detector noise and the high-frequency noise from the readout electronics).

\section{1. $\quad$ Speed considerations}

Before we present the further data reduction methods, a few words about speed considerations seem appropriate here. The lowest frequencies of the atmospheric fluctuations in Fig. 5 can be suppressed by choosing an adequate mapping speed. In the logarithmic spiral mode, a single subscan takes 36 s. Atmospheric fluctuations on longer timescales are therefore avoided. To what extent faster fluctuations degrade the image quality depends both on the source structure and on how it is sampled in the on-the-fly observing mode, scanning the source with a linear or spiral stroke pattern. The latter is used with a constant angular speed of $90^{\circ} / \mathrm{s}$, i.e., a filamentary source, repeatedly appearing in the time series of a scan, has Fourier components at a frequency of $0.25 \mathrm{~Hz}$ and its harmonics. The removal of atmospheric fluctations is therefore mandatory and will be discussed in section 3.3 . On the other hand, for a speed of $200^{\prime \prime} / \mathrm{s}$ (the largest speed occurring in the spiral stroke pattern), the main lobe of the diffraction pattern of a point-like source has a width of $8.8 \mathrm{~Hz}$ 
(FWHM) in frequency domain, which implies that the largest power is at frequencies above those of the fluctuations.

These considerations hold for Stokes I. Assuming a constant fractional polarization across the source, its modulation appears in frequency domain as a scaled version of the Stokes $I$ spectrum but now centered at the modulation frequency $f_{\mathrm{M}}$, i.e., well above the atmospheric fluctuations, which explains why they affect the polarization far less than Stokes $I$. The consequences for the demodulation will be treated in section 3.4 .

\subsection{Removal of the total power beating}

A closer inspection of the time series shows a spurious signal on top of the expected output (Fig. 6a). The spectral energy distribution (Fig. 5) confirms that it is a harmonic total power beating, starting at the fundamental frequency $f_{0}$ of the mechanical modulator rotation and visible in all the harmonics up to the Nyquist frequency. The spurious signal is not due to a gain variation and therefore independent from the total power received from the sky. Its origin can be manifold. It is common wisdom that a modulator housed in the cold part of the optics provides intrinsically more stable signals, but instability is not an issue here (the beating is a deterministic signal). The resonances that can occur in reflecting polarizers (Houde et al. 2001; Krejny et al. 2008) are also unlikely to be of concern here (the characteristics of the grid as described in section 2.4 are optimal).

Imaging the beating across the full array and as a function of time, i.e., record by record, reveals a bar-like, asymmetric total power distribution, rotating about the center of the array with the frequency $f_{0}$. The strength of this rotating feature is modulated with the frequency $2 f_{0}$ of the second harmonic. In the time series of the total power beating the combination of these spatial and temporal variations leads, for a given bolometer channel, 
to the observed profile. We also note here that the sampling frequency of $25 \mathrm{~Hz}$ is not an exact multiple of $f_{0}$ and that the spikes of the beating are not fully resolved in time; in the Fourier spectrum this leads to a further redistribution of spectral power among the harmonics. This discussion may suggest that the beating is due to the differential emissivity of the grid and the mirror of the modulator, so that the higher emissivity of the mirror leads to a net polarization perpendicular to the wires of the modulator grid. However, we cannot corroborate such a conclusion. The mirror is made from an aluminium alloy whose conductivity falls short of that of pure aluminium but can be exptected to be of the order of $\sigma=2.5 \times 10^{7} \mathrm{~S} / \mathrm{m}$, while that of tungsten is $1.8 \times 10^{7} \mathrm{~S} / \mathrm{m}$. From the Hagen-Rubens law for the spectral emissivity, $\epsilon_{\nu}=4 \sqrt{\pi \nu \epsilon_{0} / \sigma}$, and Kirchhoff's law we can then determine the expected emission. Assuming for the modulator a typical temperature of $283 \mathrm{~K}$, we obtain a polarized signal with a Rayleigh-Jeans temperature of $\sim 200 \mathrm{mK}$, accounting for the filling factor of the grid. The strength of the total power beating is comparable to that of our Orion KL data, i.e., a 100 Jy source observed with an atmospheric transmission of 55 to $72 \%$. With an aperture efficiency of 0.6 (Güsten et al. 2006), this converts to an antenna temperature of 1.4 to $1.8 \mathrm{~K}$ which is an order of magnitude above our estimate of the beating expected from a differential emissivity of mirror and grid. More dedicated measurements will be needed to clarify the origin of the beating and to further improve the system.

In practice, the total power beating dominates the signal but is strictly harmonic. In the raw data from a single scan and pixel, the modulation of the signal due to its polarization is still negligible; with the aforementioned noise-equivalent flux density, the noise in a 40 msec dump amounts to $275 \mathrm{mJy}$. In the Fourier transform of the time series the beating can be easily distinguished from the spectrum of white noise (which has a constant amplitude but a random phase) and is removed from the time series pixel-wise and scan by scan (Fig. 6b), by eliminating the narrow spikes in the Fourier spectrum of 
the adequately apodized time series, and interpolating between the real and imaginary parts next to them. In practice, this correction leads to an insignificant distortion of the observed polarization, because the latter is only piecewise periodic. Its spectral power is distributed across a much wider frequency range determined by the window function (unity when a pixel crosses the source, and zero elsewhere), especially if a filamentary structure is observed perpendicular to its long axis. Another reason for the widened Fourier spectrum of the modulation centered at $4 f_{0}$ is that the polarization vectors on the sky, while the source is scanned, either rotate with, or counter-rotate against, the motion of the waveplate. Tests with simulated sources have shown that the removal of the beating does not significantly affect the measurement of the intrinsic linear polarization. One of these test sources, a $6^{\prime}$ wide model consisting of three Gaussians, whose power is as weak as $0.5 \%$ of that of the simulated beating (100 Jy), is shown in Fig. 7. After removal of the beating and of the simulated atmospheric total power fluctuations, the intrinsic polarization of $p_{\mathrm{L}}=10 \%$ (modeled as the projection of a dipole field onto the plane of the sky) could be restored. Towards zones with a strong curvature of the field lines the $20^{\prime \prime}$ wide beam (FWHM) leads to depolarization, while an excess of the fractional polarization is observed where the Stokes $I$ emission is weak; this is due to the difficulties to conserve the largest spatial scales in the reconstruction of the data. In summary, the removal of the total power beating by the software filter described in this section shows that mounting a polarimeter in the warm part of the optics, making it more prone to resonances of this kind, can be compensated for by an adequate data reduction algorithm.

\subsection{Time series filtering}

The overlap of the near-field beam patterns of the individual pixels leads to a strong correlation between the signals received by a pair of pixels, because they "see" the same 
atmospheric fluctuations ("1/f noise"). For each data record the median signal across the array provides a good measure for the dominating contribution of the atmosphere to the received total power. However, the gains of the individual bolometer pixels, as determined from observations of Mars, Uranus or Neptune, are not appropriate because, unlike these primary calibrators, the atmospheric emission fills the entire forward-beam power pattern of each pixel. The strong correlation among the signals allows us to re-calculate and to apply the gains for the reception of the atmospheric total power. Then for each record the median signal across the array is calculated and removed from the time series, and the resulting signal is scaled to the correct far-field gain for each pixel.

While the $1 / \mathrm{f}$ noise is efficiently suppressed by means of this correlated-noise filter, the power at the highest frequencies $(f>10 \mathrm{~Hz})$ originates from the readout electronics and is suppressed by means of a wavelet filter. We refer the reader interested in wavelet filtering to appendix $\mathrm{D}$.

Both the correlated-noise and wavelet filtere may not be used for the modulated (i.e., polarized) part of the signal. Depending on the polarization structure of the observed source, the modulation leads to a partial correlation of the signals and would be distorted by the removal of correlated noise. Fortunately, such a step is not necessary here: As demonstrated in Fig. 5, the atmospheric fluctuations hardly leak into the modulation of the polarized fraction of the total power. We note, however, that although it is not modulated Stokes $I$ contaminates the polarization, due to the scanning motion. This happens, e.g., for a point source scanned at maximum speed, leading to an $8.8 \mathrm{~Hz}$ wide (FWHM) Fourier spectrum (the most extreme case demonstrated in section 3.1). The correction for this contribution in the demodulation of the polarization will be described in section 3.4 .

For the modulated, i.e., polarized fraction of the signal a frequency filter of the form

$$
g(f)=\frac{1}{2}\left(1-\cos \frac{2 \pi\left(f-f_{\text {low }}\right)}{f_{\text {high }}-f_{\text {low }}}\right) \text { for } f_{\text {low }} \leq f \leq f_{\text {high }}, g(f)=0 \text { elsewhere }
$$


is applied to the Fourier transform of suitably apodized segments of data (typically individual scans), with $f_{\text {low }}=3 \mathrm{~Hz}$ and $f_{\text {high }}=10 \mathrm{~Hz}$.

\subsection{Map-making and demodulation}

Depending on the polarization structure of the source, the phase and amplitude of the modulation changes rapidly when the telescope sweeps across the sky. Therefore, the demodulation of the data and the map-making must be performed by the same data reduction step, to retrieve Stokes $Q_{\text {eq }}$ and $U_{\text {eq }}$. The map-making, i.e., the gridding of Stokes $I$ to a regular grid, follows the usual procedure to construct a regularly sampled image from a critically, but irregularly sampled stream of data: For each pixel of the output image, only data within a cutoff radius around this pixel will be considered, and the flux assigned to this pixel is an average of this data, weighted with a convolution kernel (here a Gaussian is used). For Stokes $Q_{\text {eq }}$ and $U_{\text {eq }}$, the approach is different due to the modulation: first, the set of data located within the cutoff radius is binned into discrete waveplate position angle intervals, then this binned data is demodulated. In the logarithmic spiral mode (Siringo et al. 2009), LABOCA scans the sky at a varying speed (at constant angular velocity). Therefore the sampling of waveplate angles is no longer strictly synchronized with the spatial sampling of the area to be mapped, and the demodulation scheme needs to be generalized ${ }^{2}$. We will show now that this generalization is straightforward.

The measurement process can be represented via equation (2) as a time series, whose elements correspond to discrete time steps and therefore different waveplate angles and

\footnotetext{
${ }^{2}$ Even for the case of a regularly sampled map observed with a waveplate rotating at constant speed, for a non-zero angle of incidence the sampling of position angles cannot be strictly regular due to the projection effect.
} 
celestial positions. We write this time series as a vector $\mathcal{A}$. Since the polarization angle changes when the telescope sweeps the source, for the demodulation process we have to consider a subset of the data, $\mathcal{B} \subseteq \mathcal{A}$, whose distance from a given pixel of the output map is within the cutoff radius of the convolution kernel used in the map-making. As already mentioned, $\mathcal{B}$ may still contain a residual of the unmodulated signal, i.e., Stokes $I$. This residual is removed from $\mathcal{B}$ by a baseline subtraction, and we are left with the modulated, i.e., polarized, signal fraction only, $\boldsymbol{B}_{\mathrm{m}}$. The aim of generalized synchronous demodulation is to construct weight vectors $\boldsymbol{w}_{\mathrm{Q}}, \boldsymbol{w}_{\mathrm{U}}$, such that Stokes $Q$ and $U$ are obtained through the scalar products

$$
Q=\mathcal{B}_{\mathrm{m}} \cdot \boldsymbol{w}_{\mathrm{Q}}, U=\boldsymbol{B}_{\mathrm{m}} \cdot \boldsymbol{w}_{\mathrm{U}} .
$$

In the following we assume that PolKa is at its nominal $\lambda / 2$ tuning, and denote the corresponding sine and cosine time series as vectors with elements

$$
\mathcal{C}_{\mathrm{j}}:=\cos 4 \varphi_{\mathrm{j}}, \mathcal{S}_{\mathrm{j}}:=\sin 4 \varphi_{\mathrm{j}}
$$

where $\varphi_{\mathrm{j}}=\varphi\left(t_{\mathrm{j}}\right)$. The sensitivity of Stokes $Q$ and $U$ can then be derived from the radiometric noise $\sigma_{\text {rms }}$ in the time series $\mathcal{A}$ with

$$
\sigma_{\mathrm{Q}, \mathrm{U}}=\sigma_{\mathrm{rms}} \sqrt{\boldsymbol{w}_{\mathrm{Q}, \mathrm{U}} \cdot \boldsymbol{w}_{\mathrm{Q}, \mathrm{U}}}
$$

Because the sampling is in general irregular, we cannot expect that $\mathcal{C} \cdot \mathcal{S}=0$ (which was the prerequisite for the demodulation scheme in Siringo et al. 2004). However, generalized synchronous demodulation offers many possibilities for the construction of the weight vectors $\boldsymbol{w}_{\mathrm{Q}}, \boldsymbol{w}_{\mathrm{U}}$, also for other than sinusoidal modulations. In our case an obvious choice consists of using $\cos 4 \varphi$ and $\sin 4 \varphi$ as basis functions,

$$
\boldsymbol{w}_{\mathrm{Q}}=\mu \mathcal{C}+\nu \mathcal{S}, \boldsymbol{w}_{\mathrm{U}}=\xi \mathcal{C}+\rho \mathcal{S}
$$

and to determine the coefficients $\mu, \nu, \xi$ and $\rho$ such that equations (13) are fulfilled. This 
yields

$$
\mu=\frac{\mathcal{S}^{2}}{\mathcal{C}^{2} \mathcal{S}^{2}-(\mathcal{S} \cdot \mathcal{C})^{2}}, \quad \nu=\xi=\frac{-\mathcal{S} \cdot \mathcal{C}}{\mathcal{C}^{2} \mathcal{S}^{2}-(\mathcal{S} \cdot \mathcal{C})^{2}}, \quad \xi=\nu, \quad \rho=\frac{\mathcal{C}^{2}}{\mathcal{C}^{2} \mathcal{S}^{2}-(\mathcal{S} \cdot \mathcal{C})^{2}}
$$

Thanks to the Cauchy-Schwarz inequality the denominator of these coefficients is positive, and zero only in the case that $\mathcal{C}$ and $\mathcal{S}$ are linearly dependent, i.e., for $\tan 4 \varphi_{\mathrm{j}}=1$ or -1 for all $\varphi_{\mathrm{j}}$. The separation of Stokes $Q$ from Stokes $U$ in a technique applying a sinusoidal modulation is then impossible, but this situation is unlikely to occur, not least due to the parallactic rotation.

\section{First light observations}

\subsection{The Moon}

The lunar continuum radiation from radio to far infrared wavelengths is dominated by the thermal emission from the regolith covering the surface, originating from a frequency dependent depth of $10 \mathrm{~m}$ at $3 \mathrm{GHz}$ (the typical thickness of the regolith layer, Keihm \& Langseth 1975) to a few centimeters at $37 \mathrm{GHz}(\mathrm{Fa} \&$ Jin 2007). The Fresnel coefficients for the last refraction between the regolith and the vacuum are different for polarizations perpendicular and parallel to the plane of incidence. The resulting radial linear polarization is a useful calibration source for polarimeters. As part of the commissioning of PolKa, the Moon was observed on 2011 Dec 8, at phase 94.1\% shortly before full moon, scanning the disk in zig-zag mode twice, using different position angles for the filterwheel. The expected radial polarization pattern has been reproduced, and the fractional linear polarization amounts to up to $\sim 2 \%$ (Fig. 8). The terminator is not sharp, due to the delayed heating of the subsurface layers. The coupling of the polarized sidelobes due to the telescope's error beam pattern, irrelevant for the observations of more compact structures, leads to a slight deviation from a purely radial pattern, owing to the phase effect. 


\subsection{Tau A}

Tau A, the Crab nebula, is a plerion-type supernova remnant. The polarization of its radio emission, discovered in 1957 independently by Mayer et al. (1957) and Kuz'min \& Udal'Tsov (1959), has confirmed synchrotron radiation as the underlying mechanism. The distribution of the linear polarization across the center of the nebula, close to the pulsar which powers the synchrotron emission, is fairly smooth and the polarization angle does not vary significantly from the radio emission over visible light (Forman \& Visvanathan 1971) to X-rays (Weisskopf et al. 1978). For more recent work we refer to Hester (2008). While only upper limits have been reported for the circular polarization (at $\lambda 3 \mathrm{~mm}$, $<0.2 \%$, Wiesemeyer et al. 2011, further references therein), the linear polarization at $\lambda 3 \mathrm{~mm}$ amounts to up to $30 \%$ (Aumont et al. 2010). Our polarization map of Tau A is shown in Fig. 9, the results are summarized in Table 2 with and, for comparison, without the correction for instrumental polarization. The map was processed from 36 on-the fly maps of $150 \mathrm{sec}$ each, corresponding to an on-source observing time of $1.5 \mathrm{~h}$, at a typical atmospheric transmission of $70 \%$. The sensitivity is $\sim 20 \mathrm{mJy} /$ beam. The correction for instrumental polarization (see appendix C) changes the linear polarization by a few percent and the polarization angle by up to a few degrees. The overall agreement with other polarimetry campaigns (at $\lambda 3 \mathrm{~mm}$ with XPOL at the 30m telescope, Aumont et al. 2010, and at $\lambda 850 \mu \mathrm{m}$ with SCUPOL at the JCMT, Matthews et al. 2009) is reasonably good towards the synchrotron emission peak. The largest discrepancies occur towards the pulsar position; for the fractional polarization they are significant, but not for the polarization angles. To date it is impossible to say whether this difference is intrinsic or due to a bias introduced by the measurements and their analysis. Depolarization can be ruled out as an explanation, since SCUPOL and XPOL measure the same fractional polarization, despite their different beams (20" and $27^{\prime \prime}$ FWHM, respectively). We note that the Pulsar position is 0.5 north of the brightness peak. As mentioned in Section 2.6, the instrumental 
polarization may matter; the three polarimeters account for it in different ways: Aumont et al. (2010) applied jackknife tests to ascertain the robustness of their results, while SCUPOL applies an approximative pixel-wise correction (Greaves et al. 2003). For PolKa we refer to appendix 2.6.

Here we eventually examine whether Faraday rotation or a dust contribution to the continuum emission can rotate the polarization vector. The large-scale rotation measure towards Tau A is $\sim-21 \mathrm{rad} \mathrm{m}^{-2}$ (Bietenholz \& Kronberg 1991) and mostly external to the nebula, while in unresolved filaments of thermal gas it rises up to $300 \mathrm{rad} \mathrm{m}^{-2}$. Even in the latter medium, the differential Faraday rotation between $\lambda 3 \mathrm{~mm}$ and $\lambda 850 \mu \mathrm{m}$ is not measurable. - A dust emission component of polarized flux $P_{\text {dust }}$ and polarization angle $\psi_{\text {dust }}$ that adds to the synchrotron emission of polarized flux $P_{\text {sync }}$ and polarization angle $\psi_{\text {sync }}$ leads to a rotation $\Delta \psi$ of the polarization vector, to first order in $P_{\text {dust }} / P_{\text {sync }} \ll 1$, by

$$
\Delta \psi=\frac{P_{\text {dust }}}{2 P_{\text {sync }}} \sin \left[2\left(\psi_{\text {dust }}-\psi_{\text {sync }}\right)\right] .
$$

The largest rotation of the polarization angle occurs for dust emission polarized at $45^{\circ}$ from the synchrotron emission. Then the $\lambda 3 \mathrm{~mm}$ and $\lambda 870 \mu \mathrm{m}$ polarization angle difference of $15^{\circ}$ would require the dust emission to be polarized with $0.52 P_{\text {sync }}$ which is certainly not conceivable: Green et al. (2004) find only a small amount $\left(\lesssim 0.07 M_{\odot}, \sim 1.5 \%\right.$ of the nebula mass, Bietenholz et al. 2001) of silicate or graphite dust at a temperature of about $50 \mathrm{~K}$.

Our polarization map of Tau A is well consistent with a $32 \mathrm{GHz}$ polarization map from the Effelsberg telescope, with a similar spatial resolution (26" FWHM, Reich et al. 1998). A further comparison with a $5 \mathrm{GHz}$ VLA map (Bietenholz et al. 2001) with 1".4 resolution shows that the magnetic field orientation observed with PolKa is in the plane of the X-ray torus (Weisskopf et al. 2000). South-east of the torus, i.e. along the southern lobe of the jet, the polarization angles are similar and suggest here a magnetic field that is toroidal with respect to the jet axis. As a matter of fact, the kinked jet was successfully modeled 
by Mignone et al. (2013) assuming such a magnetic field configuration, naturally leading to the observed polarization signature. In the outer part the magnetic field structure is more complicated. Remarkably, the distribution of polarization angles (Fig. 10) shows two peaks which correspond to components that are roughly orthogonal. The peak at $160^{\circ}$ corresponds to the emission near the torus, while the other peak represents the body of the nebula. Our findings favor a scenario in which the torus is magnetically confined because plasmas with crossed magnetic fields cannot penetrate each other (Hester 2008). Furthermore, a histogram (Fig. 10) of polarization angles in the inner part of the synchrotron nebula, measured in the optical (HST/ACS, Moran et al. 2013), peaks at a polarization angle of $150^{\circ}$, which is within $10^{\circ}$ from the peak in the corresponding distribution measured with PolKa (the histogram of the optical data is wider because the sub-arcsecond resolution of the HST/ACS data traces the polarization of individual filaments). We take these correspondences as genuine pieces of evidence that PolKa reproduces the sky-plane magnetic field component of Tau A correctly, and confirms that the observed structures are magnetically controlled.

\subsection{OMC1}

The first detection of the polarization of the dust emission from the Orion Molecular Cloud I (OMC1) was made at $\lambda 270 \mu \mathrm{m}$ with the Kuiper Airborne Observatory at a spatial resolution of 90" (Hildebrand et al. 1984, further references to earlier attempts therein). Schleuning (1998) observed OMC1 at far infrared/submillimeter wavelengths $(\lambda 100 \mu \mathrm{m}$ and $\lambda 350 \mu \mathrm{m}$, respectively) across an $8^{\prime} \times 8^{\prime}$ large field. He confirmed the relatively weak linear polarization and its position angle measured by Hildebrand et al. (1984) towards the Kleinman-Low nebula (Orion KL) which is thought to be powered by an explosive event (Zapata et al. 2011), while the neighboring high mass star-forming region Orion-South and 
the envelope of Orion KL exhibit a stronger polarization. Schleuning (1998) explains the depolarization in Orion KL by the rising dust opacity towards the far-infrared, while the low polarization in the Orion Bar, a photon-dominated region seen edge-on (Tielens \& Hollenbach 1985), is attributed to a magnetic field pointing to the observer. Vaillancourt et al. (2008) studied the polarization spectrum of OMC-1 and conclude that a polarization minimum occurs between $\lambda 100 \mu \mathrm{m}$ and $\lambda 350 \mu \mathrm{m}$ while Houde et al. (2004), investigating the large scale structure of the magnetic field in Orion A, confirmed the relatively smooth polarization angle structure towards OMC1 and interprete the weak polarization levels in the Orion bar with the lower dust temperature in that region.

Our polarization map of OMC1 is shown in Fig. 11 and confirms the hourglass-like structure of the magnetic field found by the aforementioned studies. The map is obtained from a total of 54 on-the-fly scans (i.e., a total of $2.25 \mathrm{~h}$ on source), and observed with a typical atmospheric transmission of 55 to $72 \%$. The sensitivity across the map is $\sim 30 \mathrm{mJy} /$ beam. Our results agree with those from SCUPOL (Matthews et al. 2009), except for the fractional linear polarization that we detect towards OMC1-South which is as weak as in the BN/KL region where it agrees with $\operatorname{SCUPOL}\left(p_{\mathrm{L}}=0.7 \%\right)$. The polarization angles in both regions are $\sim 30^{\circ}$. We note that in general the correction for instrumental polarization improves, across the map, the agreement between our results and those of SCUPOL. The different levels of line contamination in SCUPOL and PolKa, contributing substantially to Stokes $I$ but barely to $p_{\mathrm{L}}$, may explain part of the differences. - The polarization in the filament extending northwards from Orion BN/KL is parallel to its long axis (i.e., the projected magnetic field perpendicular to it), which is consistent with previously reported results. We also note that our polarization angles agree, within the errors, with those measured by Hildebrand et al. (1984) despite the different wavelengths and spatial resolutions. We therefore confirm a fairly smooth magnetic field structure where only at smaller spatial scales $\left(<20^{\prime \prime}\right.$, i.e., $\left.0.04 \mathrm{pc}\right)$ depolarization occurs towards the 
cores of Orion KL and South. This is presumably due to either a more complex magnetic field structure there or a mixture of dust grains whose emission traces the same volume but have different properties such as temperature and size distribution, as pointed out by Vaillancourt \& Matthews (2012). Their histogram of differential polarization angles at $\lambda 350 / 850 \mu \mathrm{m}$ indeed peaks in the $0^{\circ}-10^{\circ}$ interval.

As for the Orion Bar, a prototypical photon-dominated region, we find a fractional polarization that is significantly higher, by a factor of 3 to 4 , compared to the $\mathrm{BN} / \mathrm{KL}$ and South peaks. It traces a magnetic field of which the sky-plane projection is actually along the Bar, in disagreement with the suggestion by Schleuning (1998). It is uncertain whether this result confirms the conjecture of Houde et al. (2004) that the dust temperature is lower in the Bar than in BN/KL. From their CO excitation modeling, Peng et al. (2012) infer gas temperatures in excess of $350 \mathrm{~K}$ and $250 \mathrm{~K}$ towards $\mathrm{BN} / \mathrm{KL}$ and the Bar, respectively, corroborating the $\mathrm{H}_{2}$ excitation study of Shaw et al. (2009). An in-depth discussion of these findings is beyond the scope of the work at hand and will be followed up in a forthcoming study.

\section{Conclusions and outlook}

In this work we demonstrated that PolKa, a reflection-type polarimeter installed in the warm optics of the bolometer camera LABOCA, can provide reliable measurements of the polarization of cosmic dust and synchrotron emission at submillimeter wavelengths, across fields as large as $\sim 10^{\prime}$. Similar to a classical waveplate polarimeter, the polarized fraction of the received power is detected by virtue of its modulation when the plane of polarization is continuously rotated by the waveplate. Standing waves in such a design are difficult to

suppress, especially if space limitations in the receiver cabin are an issue. However, we could show that a total power beating adding a deterministic, strictly harmonic signal to the 
modulation can be efficiently filtered out, preserving both the amplitude and phase of the modulation, and therefore the information about the intrinsic polarization. A prerequesite for this filter to work is to observe the source in the on-the-fly mode. This observing mode, with either a linear or spiral stroke pattern, is the preferred method to obtain a critically sampled map with the bolometer array whose pixels are separated by two full half-power widths. In order to demodulate the signals and to obtain maps of the Stokes parameters $Q$ and $U$ we introduced a dedicated algorithm, generalized synchronous demodulation. Moreover, a strategy to remove the spurious instrumental conversion of Stokes $I$ into Stokes $Q$ and $U$ has been proposed and successfully applied to the observations.

Our results obtained for Tau A and OMC1 agree reasonably well with previously published data, while we uncover the polarization structure of the dust emission in the Orion Bar, a prototypical photon-dominated region. This finding suggests that the projection of the magnetic field onto the plane of the sky is oriented along the Bar. The physical implications of such a pseudo-2D configuration will be discussed in a forthcoming publication. Towards the BN/KL region the polarization angles at $\lambda 870 \mu \mathrm{m}$ and $\lambda 270 \mu \mathrm{m}$ are comparable and rule out a wavelength-dependent rotation of the linear polarization. The polarization structure of the $\lambda 870 \mu \mathrm{m}$ synchroton emission from the supernova remnant Tau A confirms that its synchrotron nebula is magnetically controlled.

Meanwhile PolKa has been used to observe the polarization of molecular clouds and the star-forming regions they harbor, down to flux densities of a few $100 \mathrm{mJy}$ (e.g., Alves et al. 2014). These data demonstrate that the high sensitivity of LABOCA allows for a $3 \sigma$ detection of a fractional linear polarization of $10 \%$ in a $10^{\prime}$ wide field after 2 hours on source, under decent (0.7 mm of water vapor) yet frequent weather conditions.

We owe the APEX observatory staff a debt of gratitude. We thank Dr. S. Risse from the Fraunhofer IOF (Jena, Germany) for very fruitful discussions concerning the 
design of PolKa. The analyzer grids were donated by Manfred Tonutti (RWTH Aachen University, Germany). H.W. acknowledges helpful comments from C. Thum and insightful discussions with D. Muders, S. Heyminck, A. Lobanov and M. Houde. The referee helped to improve the paper further. - T. Hezareh's research was funded by the Alexander von Humboldt foundation. The data reduction software used the GILDAS and CFITSIO libraries (www.iram.fr/IRAMFR/GILDAS and heasarc.gsfc.nasa.gov/docs/software/). The opacity coefficients were obtained from the am model (S. Paine, SMA technical memo \#152, 2012) via the kalibrate module of the KOSMA software, Department of Physics, Cologne University. 
Table 1: Submillimeter imaging polarimeters (past and present) $)^{(a)}$.

\begin{tabular}{|c|c|c|c|c|c|c|}
\hline telescope & camera & polarimeter & $\begin{array}{c}\text { field of view }{ }^{(b)} \\
\left.{ }^{\prime}\right]\end{array}$ & $\begin{array}{c}\text { wavelength }^{(c)} \\
{[\mu \mathrm{m}]}\end{array}$ & $\begin{array}{c}\text { beam }^{(d)} \\
{\left[{ }^{\prime \prime}\right]}\end{array}$ & method \\
\hline $\mathrm{KAO}$ & \multicolumn{2}{|c|}{$\longrightarrow$ Stokes $^{(1)} \longrightarrow$} & 3.1 & 100 & 35 & quartz \\
\hline $\mathrm{CSO}$ & \multicolumn{2}{|c|}{$\longrightarrow \operatorname{Hertz}^{(2,3)}-$} & 1.8 & 360,450 & 20 & quartz \\
\hline JCMT & $\operatorname{SCUBA}^{(4)}$ & SCUPOL $^{(5)}$ & 2.3 & 450,850 & 8 & quartz \\
\hline $\mathrm{CSO}$ & SHARC-II $^{(6)}$ & $\mathrm{SHARP}^{(7)}$ & 2.4 & 350,450 & 20 & quartz \\
\hline SMTO & $\operatorname{Hertz}^{(2)}$ & $\mathrm{VPM}^{(8)}$ & 1.8 & 350 & 20 & dual VPM \\
\hline \multirow[t]{2}{*}{ Planck } & \multicolumn{2}{|c|}{$\longrightarrow \mathrm{HFI}^{(9,10)} \longrightarrow$} & n.a. & $3000,2100,1380,850$ & 282 & PSB \\
\hline & \multicolumn{2}{|c|}{ - BLAST-Pol ${ }^{(11)}$} & $13.5 \times 6.5$ & $250,350,500$ & 30 & PSB \\
\hline APEX & $\mathrm{LABOCA}^{(12)}$ & $\mathrm{PolKa}^{(13)}$ & 12.3 & 870 & 20 & $\mathrm{RPM}$ \\
\hline
\end{tabular}

Note. - (a) Cameras are specified separately when subsequently equipped with a polarimeter. Only commissioned, published systems are considered. (b) Largest dimension of instantaneous field-of-view (refers to the shortest wavelength for detectors with several bands). (c) Central wavelength of camera bandpass. (d) FWHM of main beam in Stokes $I$, not necessarily the diffraction limit of the telescope. Refers to shortest wavelength for polarimeters with several bands. References: (1) Platt et al. (1991), (2) Schleuning et al. (1997), (3) Dowell et al. (1998), (4) Holland et al. (1999), (5) Greaves et al. (2003), (6) Dowell et al. (2003), (7) Li et al. (2008), (8) Krejny et al. (2008), (9) Lamarre et al. (2010), (10) Rosset et al. (2010), (11) Fissel et al. (2010), (12) Siringo et al. (2009), (13) Siringo et al. (2012).

Acronyms: APEX - Atacama Pathfinder Experiment (Llano Chajnantor, Chile), BLAST-Pol - Balloonborne Large Aperture Submillimeter Telescope for Polarimetry, CSO - Caltech Submillimeter Observatory (Mauna Kea, Hawaii), HFI - High Frequency Instrument, JCMT - James Clerk Maxwell Telescope (Mauna Kea, Hawaii), KAO - Kuiper Airborne Observatory, LABOCA - Large Apex Bolometer Camera, PolKa Polarimeter für Bolometerkameras, PSB - polarization sensitive bolometer elements, RPM - reflecting polarization modulator, SCUBA - Submillimeter Common-User Bolometer Array, SHARC - Submillimeter High Angular Resolution Camera, SHARP - Submillimeter High Angular Resolution Polarimeter, SMTO - Submillimeter Telescope Observatory (Mount Graham, Arizona), VPM - variable delay polarization modulator. 
Table 2: Synoptic summary of results.

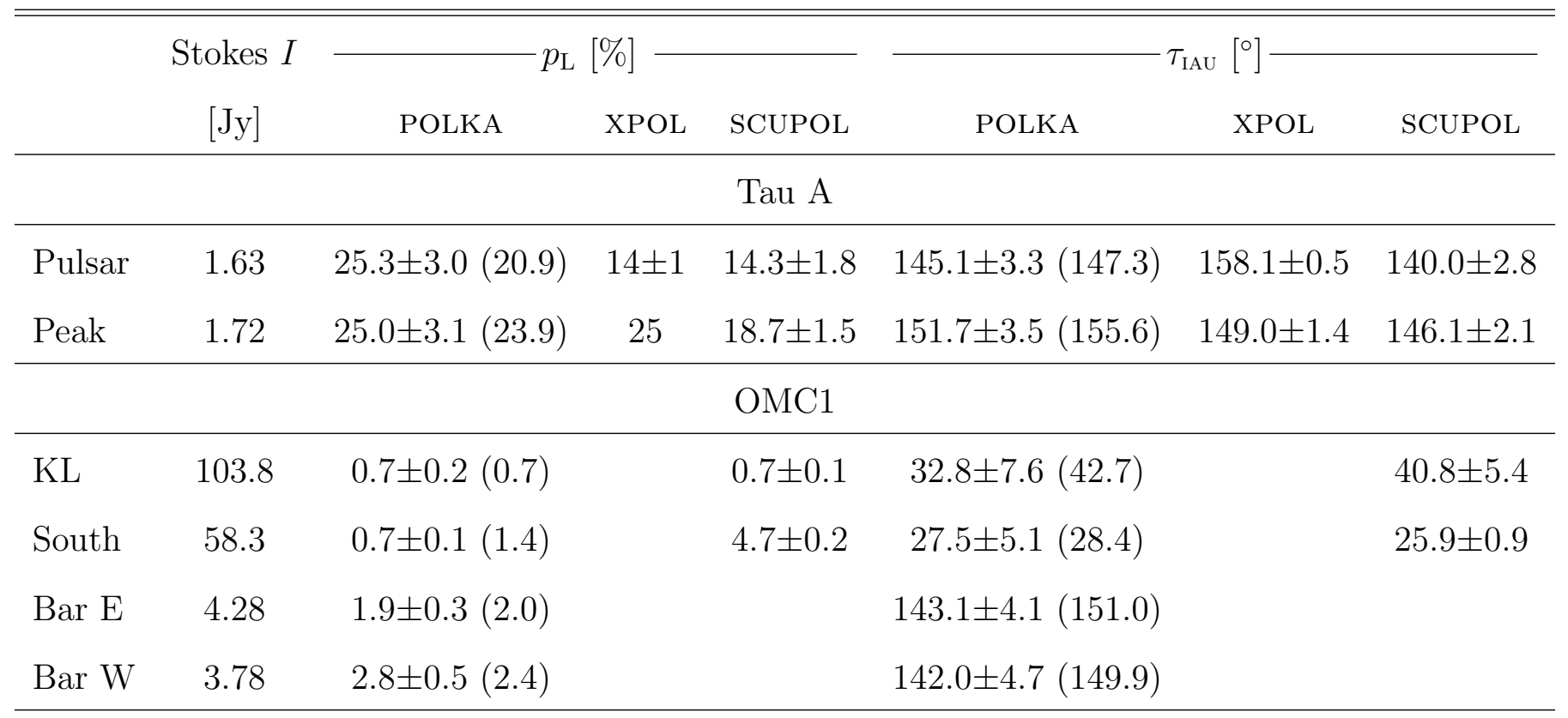

Note. - The PolKa data is corrected for instrumental polarization (uncorrected values are given in brackets). Flux densities refer to a $20^{\prime \prime}$ beam (FWHM), errors in Stokes $I$ are dominated by systematics. The position of Orion KL is $\alpha_{\mathrm{J} 2000}=05: 35: 14.283, \delta_{\mathrm{J} 2000}=-05: 22: 31.32$, in Tau A the pulsar is at $\alpha_{\mathrm{J} 2000}=05: 34: 31.938, \delta_{\mathrm{J} 2000}=+22: 00: 52.18$. Results from 30m/XPOL (Aumont et al. 2010, 27" FWHM) and JCMT/SCUPOL (Matthews et al. 2009, 20" FWHM) are shown for comparison. 


\section{REFERENCES}

Aitken, D. K., Hough, J. H., \& Chrysostomou, A. 2006, MNRAS, 366, 491

Alves, F., Frau, P., Girart, J. M., et al. 2014, A\&A in press, arXiv:1408.5133

André, P., Men'shchikov, A., Bontemps, S., et al. 2010, A\&A, 518, L102

Aumont, J., Conversi, L., Thum, C., et al. 2010, A\&A, 514, A70

Bietenholz, M. F., Frail, D. A., \& Hester, J. J. 2001, ApJ, 560, 254

Bietenholz, M. F., \& Kronberg, P. P. 1991, ApJ, 368, 231

Born, M., \& Wolf, E. 1999, Principles of Optics, Cambridge, UK: Cambridge University Press, October 1999.

Butler, B.J. 2008, http://www.aoc.nrao.edu/ bbutler/work/mars/model/

Chambers, W. G., Costley, A. E., \& Parker, T. J. 1988, International Journal of Infrared and Millimeter Waves, 9, 157

Chambers, W. G., Parker, T. J., \& Costley, A.E. 1986, in Infrared and Millimeter Waves (ed. K. J. Button), 16, 77

Chambers, W. G., Mok, C. L., \& Parker, T. J. 1980, Journal of Physics A Mathematical General, 13, 1433

Chandrasekhar, S., \& Fermi, E. 1953, ApJ, 118, 113

Chu, T. S., Gans, M. J., \& Legg, W. E. 1975, AT T Technical Journal, 54, 1665

Chuss, D. T., Wollack, E. J., Henry, R., et al. 2012a, Appl. Opt., 51, 197

Chuss, D. T., Wollack, E. J., Pisano, G., et al. 2012b, Appl. Opt., 51, 6824 
Chuss, D. T., Wollack, E. J., Moseley, S. H., \& Novak, G. 2006, Appl. Opt., 45, 5107

Crutcher, R. M. 2012, ARA\&A, 50, 29

Davis, L., Jr., \& Greenstein, J. L. 1951, ApJ, 114, 206

Daubechies, I. 1992, CBMS-NSF regional conference series in applied mathematics, Lectures delivered at the CBMS conference on wavelets, University of Lowell, Mass., June 1990, Philadelphia: Society for Industrial and Applied Mathematics (SIAM), 1992

Dowell, C. D., Allen, C. A., Babu, R. S., et al. 2003, Proc. SPIE, 4855, 73

Dowell, C. D., Hildebrand, R. H., Schleuning, D. A., et al. 1998, ApJ, 504, 588

Draine, B. T., \& Fraisse, A. A. 2009, ApJ, 696, 1

Draine, B. T. 2004, The Cold Universe, Saas-Fee Advanced Course 32, Springer-Verlag, 308 pages, 129 figures, Lecture Notes 2002 of the Swiss Society for Astronomy and Astrophysics (SSAA), Springer, 2004. Edited by A.W. Blain, F. Combes, B.T. Draine, D. Pfenniger and Y. Revaz, ISBN 354040838x, p. 213

Draine, B. T., \& Weingartner, J. C. 1997, ApJ, 480, 633

Draine, B. T., \& Weingartner, J. C. 1996, ApJ, 470, 551

Fa, W., \& Jin, Y.-Q. 2007, Icarus, 190, 15

Fano, U. 1954, Physical Review, 93, 121

Fissel, L. M., Ade, P. A. R., Angilè, F. E., et al. 2010, Proc. SPIE, 7741,

Forman, W., \& Visvanathan, N. 1971, Nature, 229, 39

Girart, J. M., Frau, P., Zhang, Q., et al. 2013, ApJ, 772, 69 
Greaves, J. S., Holland, W. S., Jenness, T., et al. 2003, MNRAS, 340, 353

Green, D. A., Tuffs, R. J., \& Popescu, C. C. 2004, MNRAS, 355, 1315

Groesbeck, T. D. 1995, Ph.D. Thesis

Guan, X., Stutzki, J., Graf, U. U., et al. 2012, A\&A, 542, L4

Güsten, R., Nyman, L. Å., Schilke, P., et al. 2006, A\&A, 454, L13

Hamaker, J. P., Bregman, J. D., \& Sault, R. J. 1996, A\&AS, 117, 137

Heitsch, F., Burkert, A., Hartmann, L. W., Slyz, A. D., \& Devriendt, J. E. G. 2005, ApJ, 633, L113

Heitsch, F., Zweibel, E. G., Mac Low, M.-M., Li, P., \& Norman, M. L. 2001, ApJ, 561, 800

Hennebelle, P., Banerjee, R., Vázquez-Semadeni, E., Klessen, R. S., \& Audit, E. 2008, A\&A, 486, L43

Hester, J. J. 2008, ARA\&A, 46, 127

Hezareh, T., Wiesemeyer, H., Houde, M., Gusdorf, A., \& Siringo, G. 2013, A\&A, 558, A45

Hildebrand, R. H., Kirby, L., Dotson, J. L., Houde, M., \& Vaillancourt, J. E. 2009, ApJ, 696,567

Hildebrand, R. H., Dragovan, M., \& Novak, G. 1984, ApJ, 284, L51

Hiltner, W. A. 1951, ApJ, 114, 241

Holland, W. S., Robson, E. I., Gear, W. K., et al. 1999, MNRAS, 303, 659

Houde, M., Vaillancourt, J. E., Hildebrand, R. H., Chitsazzadeh, S., \& Kirby, L. 2009, ApJ, 706,1504 
Houde, M., Dowell, C. D., Hildebrand, R. H., et al. 2004, ApJ, 604, 717

Houde, M., Akeson, R. L., Carlstrom, J. E., et al. 2001, PASP, 113, 622

Howard, J., Peebles, W.A., \& Luhman, N.C. 1986, IJIMW, 7, 1591

Johnstone, D., \& Bally, J. 1999, ApJ, 510, L49

Jones, R. C. 1941, Journal of the Optical Society of America (1917-1983), 31, 488

Keating, B.G. in: Ade, P. A. R., Chuss, D. T., Hanany, S., et al. 2009, Journal of Physics Conference Series, 155, 012006, pp. 11-21

Keihm, S. J., \& Langseth, M. G. 1975, Icarus, 24, 211

Khazan, M. A. 2002, PhD thesis, Univ. Hamburg, http://www.physnet.unihamburg.de/services/biblio/dissertation.htm

Kim, S.-H., \& Martin, P. G. 1995, ApJ, 444, 293

Krejny, M., Chuss, D., D’Aubigny, C. D., et al. 2008, Appl. Opt., 47, 4429

Kuo, C. L., Bock, J. J., Bonetti, J. A., et al. 2008, Proc. SPIE, 7020

Kuz'min, A. D., \& Udal'Tsov, V. A. 1959, Soviet Ast., 3, 39

Lamarre, J.-M., Puget, J.-L., Ade, P. A. R., et al. 2010, A\&A, 520, A9

Lamb, H. 1898, Proc.Lond.Math.Soc. 29, 523

Lazarian, A., \& Hoang, T. 2011, Astronomical Society of the Pacific Conference Series, 449, 116

Lazarian, A. 2007, J. Quant. Spec. Radiat. Transf., 106, 225

Lazarian, A., \& Hoang, T. 2007, ApJ, 669, L77 
Leahy, J. P., Bersanelli, M., D’Arcangelo, O., et al. 2010, A\&A, 520, A8

Lellouch, E., \& Amri, H. 2006, http://www.lesia.obspm.fr/perso/emmanuel-lellouch/mars

Li, H., Dowell, C. D., Kirby, L., Novak, G., \& Vaillancourt, J. E. 2008, Appl. Opt., 47, 422

Marsden, P. L., Gillett, F. C., Jennings, R. E., et al. 1984, ApJ, 278, L29

Matthews, B. C., McPhee, C. A., Fissel, L. M., \& Curran, R. L. 2009, ApJS, 182, 143

Matsumura, M., \& Bastien, P. 2009, ApJ, 697, 807

Mayer, C. H., McCullough, T. P., \& Sloanaker, R. M. 1957, ApJ, 126, 468

Mignone, A., Striani, E., Tavani, M., \& Ferrari, A. 2013, MNRAS, 436, 1102

Moran, P., Shearer, A., Mignani, R. P., et al. 2013, MNRAS, 433, 2564

Motte, F., Zavagno, A., Bontemps, S., et al. 2010, A\&A, 518, L77

Mouschovias, T. C., \& Ciolek, G. E. 1999, NATO ASIC Proc. 540: The Origin of Stars and Planetary Systems, 305

Moyerman, S., Bierman, E., Ade, P. A. R., et al. 2013, ApJ, 765, 64

Muders, D., Hafok, H., Wyrowski, F., et al. 2006, A\&A, 454, L25

Orton, G. S., Fletcher, L. N., Moses, J. I., et al. 2014, submitted to Icarus

Peng, T.-C., Wyrowski, F., Zapata, L. A., Güsten, R., \& Menten, K. M. 2012, A\&A, 538, A12

Platt, S. R., Hildebrand, R. H., Pernic, R. J., Davidson, J. A., \& Novak, G. 1991, PASP, 103,1193 
Press, W. H., Teukolsky, S. A., Vetterling, W. T., \& Flannery, B. P. 1992, Numerical Recipes in C, 2nd ed., Cambridge University Press, 1992, p. 591

Prigent, C., Abba, P., \& Cheudin, M. 1988, IJIMW, 9, 477

Purcell, E. M. 1979, ApJ, 231, 404

Reich, W., Fürst, E., \& Kothes, R. 1998, Mem. Soc. Astron. Italiana, 69, 933

Rosset, C., Tristram, M., Ponthieu, N., et al. 2010, A\&A, 520, A13

Rudy, D. J., Muhleman, D. O., Berge, G. L., Jakosky, B. M., \& Christensen, P. R. 1987, Icarus, 71,159

Salter, C. J., Reynolds, S. P., Hogg, D. E., Payne, J. M., \& Rhodes, P. J. 1989, ApJ, 338, 171

Schleuning, D. A. 1998, ApJ, 493, 811

Schleuning, D. A., Dowell, C. D., Hildebrand, R. H., Platt, S. R., \& Novak, G. 1997, PASP, 109,307

Shaw, G., Ferland, G. J., Henney, W. J., et al. 2009, ApJ, 701, 677

Shinnaga, H., Tsuboi, M., \& Kasuga, T. 1999, PASJ, 51, 175

Siringo, G., Kovács, A., Kreysa, E., et al. 2012, Proc. SPIE, 8452

Siringo, G., Kreysa, E., Kovács, A., et al. 2009, A\&A, 497, 945

Siringo, G., Kreysa, E., Reichertz, L. A., \& Menten, K. M. 2004, A\&A, 422, 751

Thum, C., Wiesemeyer, H., Paubert, G., Navarro, S., \& Morris, D. 2008, PASP, 120, 777

Tielens, A. G. G. M., \& Hollenbach, D. 1985, ApJ, 291, 747 
Vaillancourt, J. E., \& Matthews, B. C. 2012, ApJS, 201, 13

Vaillancourt, J. E., Dowell, C. D., Hildebrand, R. H., et al. 2008, ApJ, 679, L25

Wardle, J. F. C., \& Kronberg, P. P. 1974, ApJ, 194, 249

Weisskopf, M. C., Hester, J. J., Tennant, A. F., et al. 2000, ApJ, 536, L81

Weisskopf, M. C., Silver, E. H., Kestenbaum, H. L., Long, K. S., \& Novick, R. 1978, ApJ, 220, L117

Wiesemeyer, H., Thum, C., Morris, D., Aumont, J., \& Rosset, C. 2011, A\&A, 528, A11

Zapata, L. A., Schmid-Burgk, J., \& Menten, K. M. 2011, A\&A, 529, A24

\section{A. Polarimetry in coherency matrix formulation}

The transfer of a radiation field of mixed polarization through a series of optical devices can be conveniently described in the framework of the Jones calculus in a $\mathbb{C}_{2}$ vector space (Jones 1941) in combination with the coherency matrix formulation used in modern optics (e.g., Born \& Wolf 1999). The description of ensembles in quantum theory lends itself to the introduction of the Stokes parameters as coefficients appearing in the expansion of the coherency matrix by the identity matrix and the three Pauli spin matrices (Fano 1954): In quantum-electrodynamics there are two probabilities to consider, namely, the probability for a photon to be in a given polarization state, and the probability for this polarization state to be represented in an ensemble of photons. The density matrix reads

$$
\boldsymbol{\rho}=\frac{1}{2}\left(\begin{array}{cc}
I+Q & U-i V \\
U+i V & I-Q
\end{array}\right) .
$$

This manuscript was prepared with the AAS IATEX macros v5.2. 
In wave optics, the Stokes parameters $I, Q, U$ and $V$ would be replaced by the corresponding integrals of Kirchhoff's diffraction formula. Subsequent reflections and rotations of the plane of incidence are then decribed by a series of similarity transformations $\mathbf{T}=\mathbf{T}_{\mathbf{1}} \cdot \mathbf{T}_{\mathbf{2}} \cdot \mathbf{T}_{\mathbf{3}}$ etc. such that

$$
\rho^{\prime}=\mathrm{T} \rho \mathrm{T}^{-1}
$$

Like in quantum theory, the measurement is described by a projection operator, i.e., an outer vector product

$$
\mathbf{A}=\left(\begin{array}{c}
g_{\mathrm{x}} \\
g_{\mathrm{y}}
\end{array}\right) \cdot\left(g_{\mathrm{x}}^{*}, g_{\mathrm{y}}^{*}\right)=\left(\begin{array}{cc}
\left|g_{\mathrm{x}}\right|^{2} & g_{\mathrm{x}} g_{\mathrm{y}}^{*} \\
g_{\mathrm{x}}^{*} g_{\mathrm{y}} & \left|g_{\mathrm{y}}\right|^{2}
\end{array}\right)
$$

where $g_{\mathrm{x}}$ and $g_{\mathrm{y}}$ are the complex gain factors for the signal detection in horizontal, respectively vertical, polarization. The ensemble average $S$, i.e., the recorded signal, can be shown to be

$$
S=\operatorname{tr}(\boldsymbol{\rho} \mathbf{A})
$$

which yields for, e.g., a receiver detecting horizontal polarization with vanishing cross-polarization $\left(g_{\mathrm{y}}=0\right), S=g_{\mathrm{x}}(I+Q) / 2$, as expected.

The coherency matrix formalism will now be applied to the measurement equation for

a reflection-type polarimeter. Hamaker et al. (1996) also combine the Jones and coherency matrix calculus but follow a mathematically different approach. It can be shown that their description is formally equivalent to the treatment used here but the introduction of the projection operator may be more intuitive for the understanding of the underlying physical processes, since we consider a detector as a filter for a given polarization state.

\section{B. Derivation of the measurement equation}

A half-waveplate rotates the plane of polarization by an angle $2 \varphi$ without rotating the field of view. The corresponding transformation can therefore be written as a similarity 
transformation of the coherency matrix as described in the previous section. First, we rotate the coordinate system by an angle $\varphi$ such that the vertical axis of the new system is along the wires, i.e.,

$$
\mathbf{T}_{1}=\left(\begin{array}{cc}
\cos \varphi & \sin \varphi \\
-\sin \varphi & \cos \varphi
\end{array}\right)
$$

The next transformation describes the reflection off the wires of the vertically polarized component (the first term on the r.h.s.), while the horizontally polarized component is transmitted and reflected by the mirror, with a phase shift $\Phi$ (the second term):

$$
\mathbf{T}_{2}=\left(\begin{array}{ll}
0 & 0 \\
0 & 1
\end{array}\right)+\left(\begin{array}{cc}
\exp i \Phi & 0 \\
0 & 0
\end{array}\right)
$$

Rotating back the coordinate system to the original orientation, and accounting for the reflection of both polarization components is achieved by

$$
\mathbf{T}_{3}=\left(\begin{array}{rr}
-\cos \varphi & \sin \varphi \\
\sin \varphi & \cos \varphi
\end{array}\right)
$$

Applying the transformation $\mathbf{T}=\mathbf{T}_{3} \mathbf{T}_{2} \mathbf{T}_{1}$ to the density matrix equation $\mathrm{A2}$ yields (note that the reflection of both polarization components changes the signs of Stokes $U$ and $V$ )

$$
\boldsymbol{\rho}^{\prime}=\frac{1}{2}\left(\begin{array}{rr}
I+Q^{\prime} & -U^{\prime}+i V^{\prime} \\
-U^{\prime}-i V^{\prime} & I-Q^{\prime}
\end{array}\right)
$$

with

$$
Q^{\prime}=Q\left(\cos ^{2} 2 \varphi+\sin ^{2} 2 \varphi \cos \Phi\right), U^{\prime}=\frac{1}{2} U(1-\cos \Phi) \sin 4 \varphi, V^{\prime}=\frac{1}{2} \sin 2 \varphi \sin \Phi
$$

If the wires of the analyzer grid are oriented vertically, then horizontal polarization is transmitted and detected, i.e., the projection operator equation A3 reads, for 100\% transmission,

$$
\mathbf{A}=\left(\begin{array}{ll}
1 & 0 \\
0 & 0
\end{array}\right)
$$


and equation A4 yields $S=\operatorname{tr}\left(\boldsymbol{\rho}^{\prime} \mathbf{A}\right)=\boldsymbol{\rho}_{11}^{\prime}$.

The beam is folded several times on its way from the focal plane to the camera. The corresponding transformation can be included as a series of reflections, each described by a transformation

$$
\mathbf{T}_{\mathrm{r}}=\left(\begin{array}{rr}
-\cos \Theta & -\sin \Theta \\
-\sin \Theta & \cos \Theta
\end{array}\right)
$$

where $\Theta$ is the position angle of the axis about which the mirror is tilted (with respect to the $x$ axis in the Cassegrain coordinate system, Fig. 3). In Eqs. (B5) this transformation leads to the substitution $\varphi \rightarrow \varphi-\Theta / 2$ and then, by virtue of equation (A4), to the result given by equation (2).

\section{Correction for instrumental polarization}

In the following algorithm we assume that the Stokes parameters are linear, i.e., the instrumental polarization adds to the intrinsic one. We neglect the spurious conversion among the Stokes parameters $Q$ and $U$, which is caused by the error in the determination of the orientation of the analyzer grid. In the following, $S_{0}, S_{1}, S_{2}$ and $S_{3}$ denote the brightness distribution of the Stokes parameters $I, Q, U$ and $V$ on the sky. $P_{1}, P_{2}$ and $P_{3}$ describe the power pattern of the instrumental conversion from Stokes $I$ into Stokes $Q, U$ and $V$, respectively, whereas $P_{0}$ is power beam pattern of the antenna.

The ideal response of the telescope and its optics in the receiver cabin would be

$$
F_{j}=S_{j} * P_{0} \text { for } j=0 \text { to } 3
$$

such that the $F_{\mathrm{j}}$ are main-beam calibrated flux densities of the Stokes parameters. In reality, the observations yield rather

$$
F_{0, \mathrm{obs}}=F_{0}, F_{\mathrm{j}, \mathrm{obs}}=S_{\mathrm{j}} * P_{0}+S_{0} * P_{\mathrm{j}} \text { for } j=1,2,3
$$


All quantities in this set of equations are functions of offsets in the plane tangential to the celestial sphere, i.e., $(\Delta \alpha \cos \delta, \Delta \delta)$, and $*$ is the convolution product. The basic idea of the correction procedure is that the response functions $P_{1}, P_{2}$ and $P_{3}$ can be measured on a spatially unresolved, unpolarized calibrator, e.g., Uranus, Mars or Mercury (the latter two are intrinsically weakly linearly polarized but the radial orientation of polarization vectors leads to a mutual cancellation within the telescope's main beam; Mercury should be observed at full phase). Such "Stokes beam maps", sampled with the same mapping procedure as the maps to be corrected, i.e., on-the-fly maps with a spiral or linear stroke pattern, yield

$$
F_{\mathrm{j}, \mathrm{cal}}=\Pi * P_{\mathrm{j}} \text { for } j=1,2,3
$$

where the indices $j$ stand again for the Stokes parameters $Q, U$ and $V$. The following analysis is computationally easier to perform in Fourier space (no information will be lost, provided that aliasing in the discrete fast Fourier transform is avoided by using sufficiently large maps or, if the observed emission does not fall to zero within the map, to apply an apodization). The 2D Fourier transforms, as a function of their spatial frequencies, are denoted $\hat{F}_{\mathrm{j}}, \hat{P}_{\mathrm{j}}$ for $j=0,1,2,3$. From the Stokes $I$ image of our source and the "Stokes beams" we can model the instrumental polarization

$$
\hat{F}_{\mathrm{j}, \text { mod }}=\hat{F}_{0} \cdot \hat{F}_{\mathrm{j}, \text { cal }} \text { for } j=1,2,3
$$

and reconstruct $Q$ and $U$ from the Fourier transform of

$$
\hat{F}_{\mathrm{j}} \cdot \hat{\Pi} \cdot \hat{P}_{0}=\hat{F}_{\mathrm{j}, \mathrm{obs}} \cdot \hat{\Pi} \cdot \hat{P}_{0}-\hat{F}_{\mathrm{j}, \bmod }
$$

We note that while the correction is done at a reduced spatial resolution, it is possible to recover the original resolution by dividing by $\hat{P} i \cdot \hat{P}_{0}$ up to a reasonable cutoff of spatial frequencies, and transforming the resulting $\hat{F}_{\mathrm{j}}$ back to the sky plane. This means that we can only correct for the effects of instrumental polarization down to spatial scales which 
are a factor $\sqrt{2}$ larger than the original resolution. Only an interferometer map of $F_{0}$, with an order of magntitude better resolution, can avoid this limitation (provided that the interferometer data are corrected for the missing short spatial frequencies).

Our algorithm has been demonstrated with the test source shown in Fig. 7 and also with XPOl data from the 30m telescope (Hezareh et al. 2013). As expected, the correction is largest at the edges of sources with a nearby strong emission peak, because the Stokes beams $P_{\mathrm{j}}(j=1,2,3)$ are usually wider than the antenna power beam pattern $P_{0}$, resulting in an increase of the fractional instrumental polarization. This is often a direct consequence of the fact that the design of telescopes and their tertiary optics is optimized for Stokes $I$ but not for the other Stokes parameters.

\section{Low-pass wavelet filter}

The properties of the discrete wavelet transformation (hereafter DWT, see e.g., Press et al. 1992) has properties that are similar to those of the fast Fourier transform, e.g., the basis functions of both linear transformations are localized in frequency space. The basis functions of the DWT, however, are also localized in the time domain.

Here we use the DWT to filter out the high-frequency noise in the time series of signals measured by a bolometer pixel, applying Daubechies wavelet functions with up to 12 coefficients (Daubechies 1992). We perform the DWT, identify the contribution of the high-frequency noise (contained in the high wavelet numbers), set it to zero, and transform back to the time domain. Fig. 12 shows a demonstration where only up to a quarter of the total number $\left(2^{13}\right)$ of wavelets is retained and transformed back. The spectral analysis of the time series before and after the wavelet filtering shows that the high frequency noise $(f>8 \mathrm{~Hz})$ is efficiently suppressed, while the intrinsic profile of the source is preserved. 


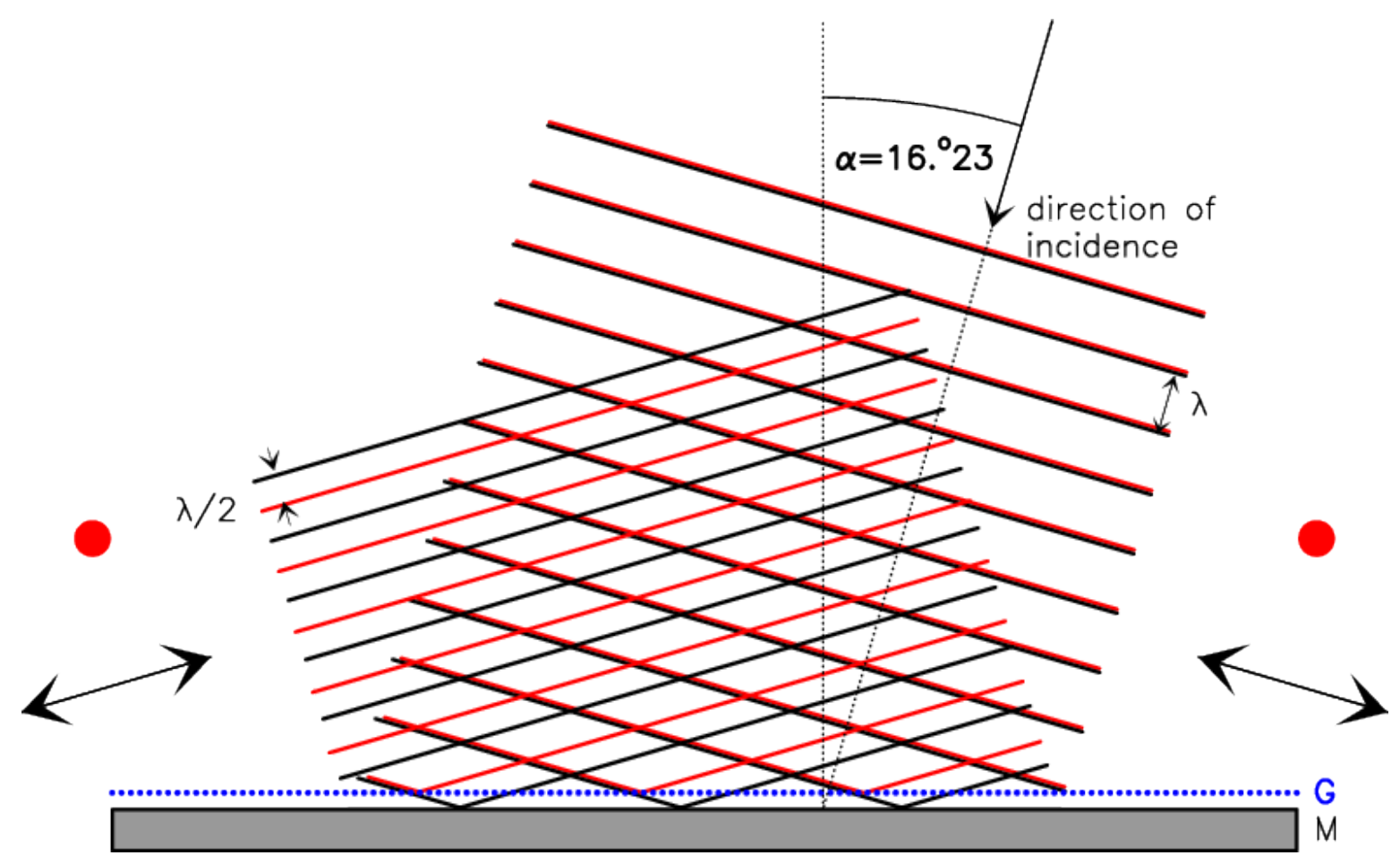

Fig. 1.- Demonstration of wave propagation in a reflection-type polarimeter with $\lambda / 2$ tuning. The wave component with polarization in the drawing plane is shown as black lines (representing the maxima of the wave front, transmitted by grid $\mathrm{G}$ ), that with polarization perpendicular to the drawing plane as red lines (reflected by grid $\mathrm{G}$ ). A pictorial representation of these polarizations is also shown, for the incident wave (right) and the reflected one (left). The grid $(\mathrm{G})$ is shown by blue dots (wires perpendicular to the drawing plane), and, at the bottom, the mirror (M) in gray. Not to scale. The angle of incidence is given as for the installation of PolKa in the Cassegrain cabin of the APEX telescope. 

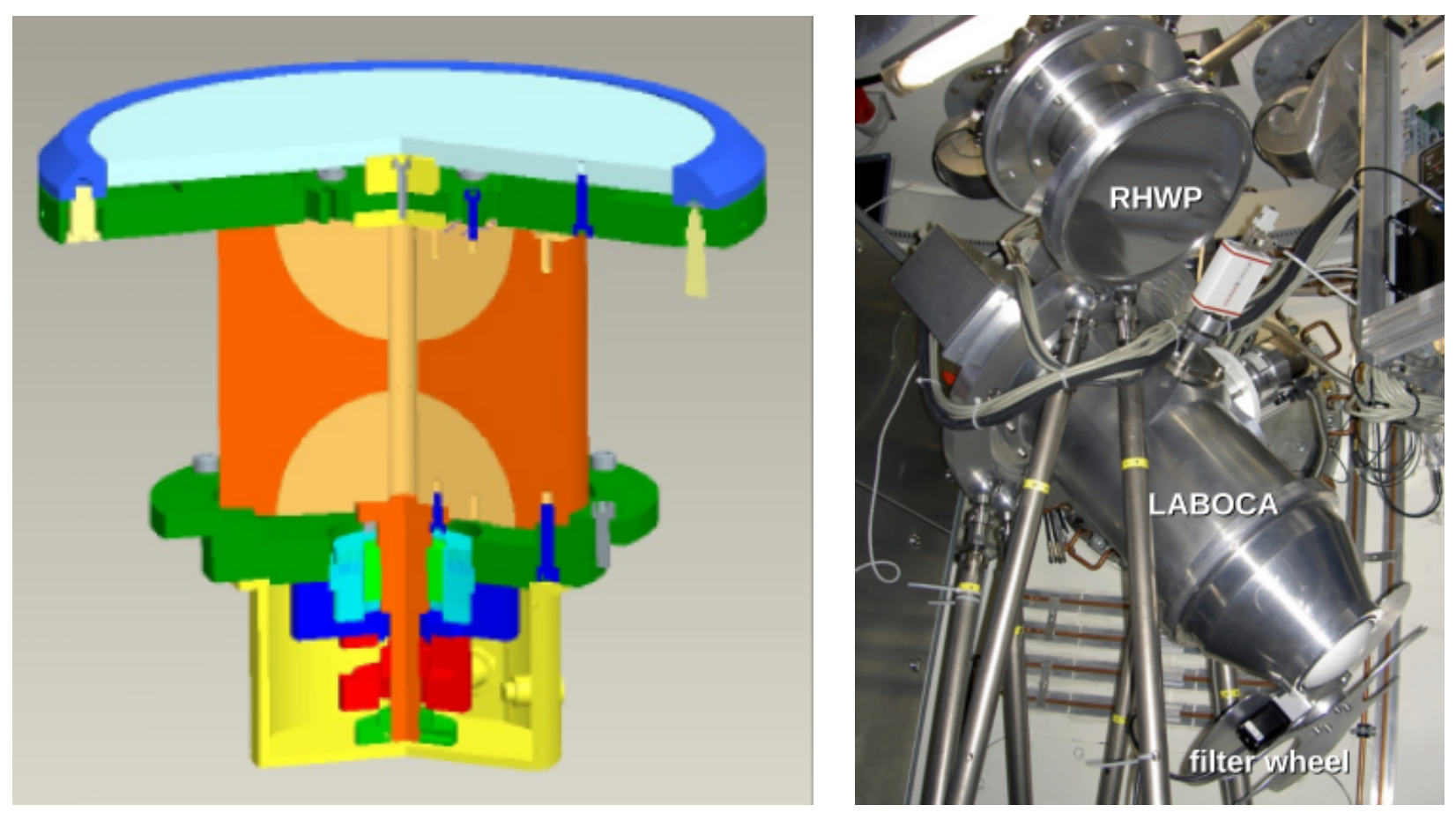

Fig. 2.- Left: Design drawing of PolKa (reproduced with permission from Dr. Stefan Risse; copyright Fraunhofer IOF). The air bearing consists of the two hemispheres (shown in pale orange). The frame of the grid and the mirror on which it is mounted by means of three micrometer screws are shown in azure and bright blue, respectively. Right: Installation of PolKa in the Cassegrain cabin of the APEX telescope. The various devices are labelled in the photo (RHWP stands for reflecting half-waveplate). 


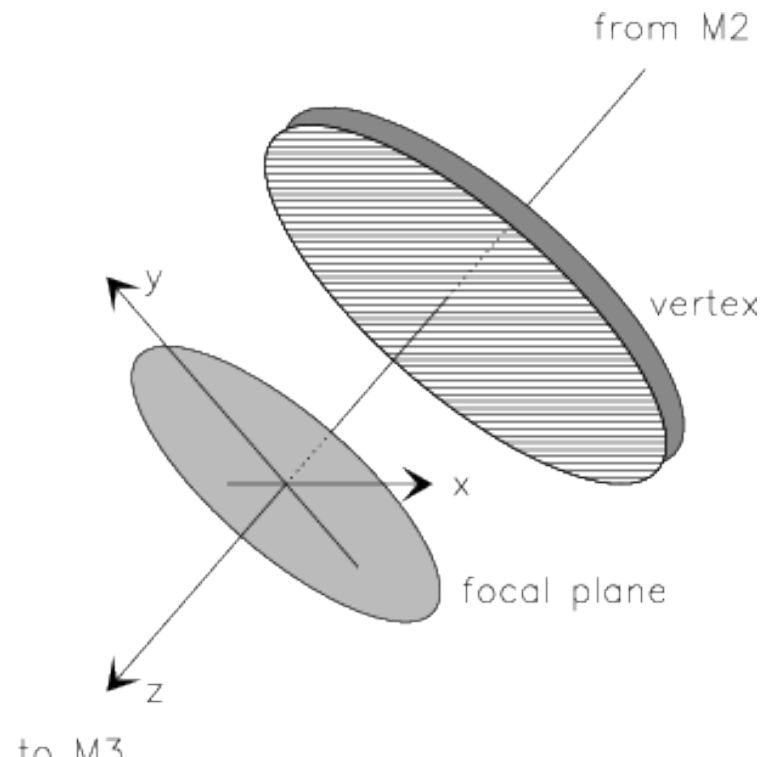

Fig. 3.- Schematic drawing of the Cassegrain coordinate system (not to scale), for $45^{\circ}$ elevation. The hatched area shows the vertex, the gray area the focal plane. The directions from the secondary mirror $\left(M_{2}\right)$ and to the tertiary mirror $\left(M_{3}\right)$ are also indicated. The $\mathrm{x}$-axis is parallel to the elevation axis of the telescope. Owing to the image inversion in the focal plane, the $\mathrm{y}$-axis points towards the horizon and the $\mathrm{x}$-axis to the west when the antenna is pointed to north. 


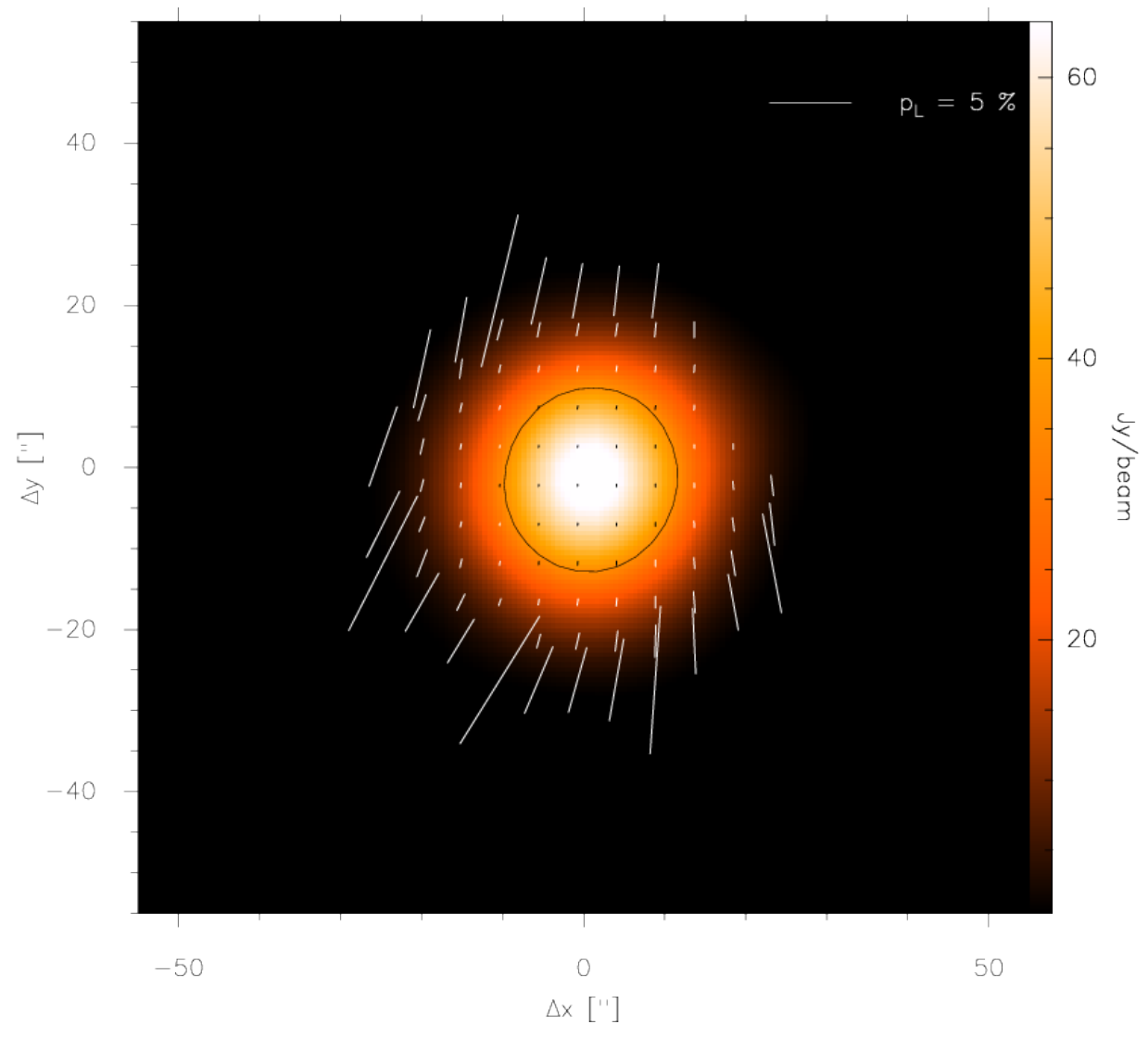

Fig. 4. - Stokes $I$ image of Uranus (color scale as given by wedge to the right-hand side). Vectors of the instrumental polarization are overlaid, as defined in the Cassegrain reference frame (counting the polarization angle ccw from the positive x-axis, cf. Fig. 3). A polarization of $5 \%$ is indicated in the upper right-hand corner. The black contour is at the half-maximum level of Stokes $I$. Only polarizations with $p_{\mathrm{L}} \geq 3 \sigma_{\mathrm{p}_{\mathrm{L}}}$ are shown. 


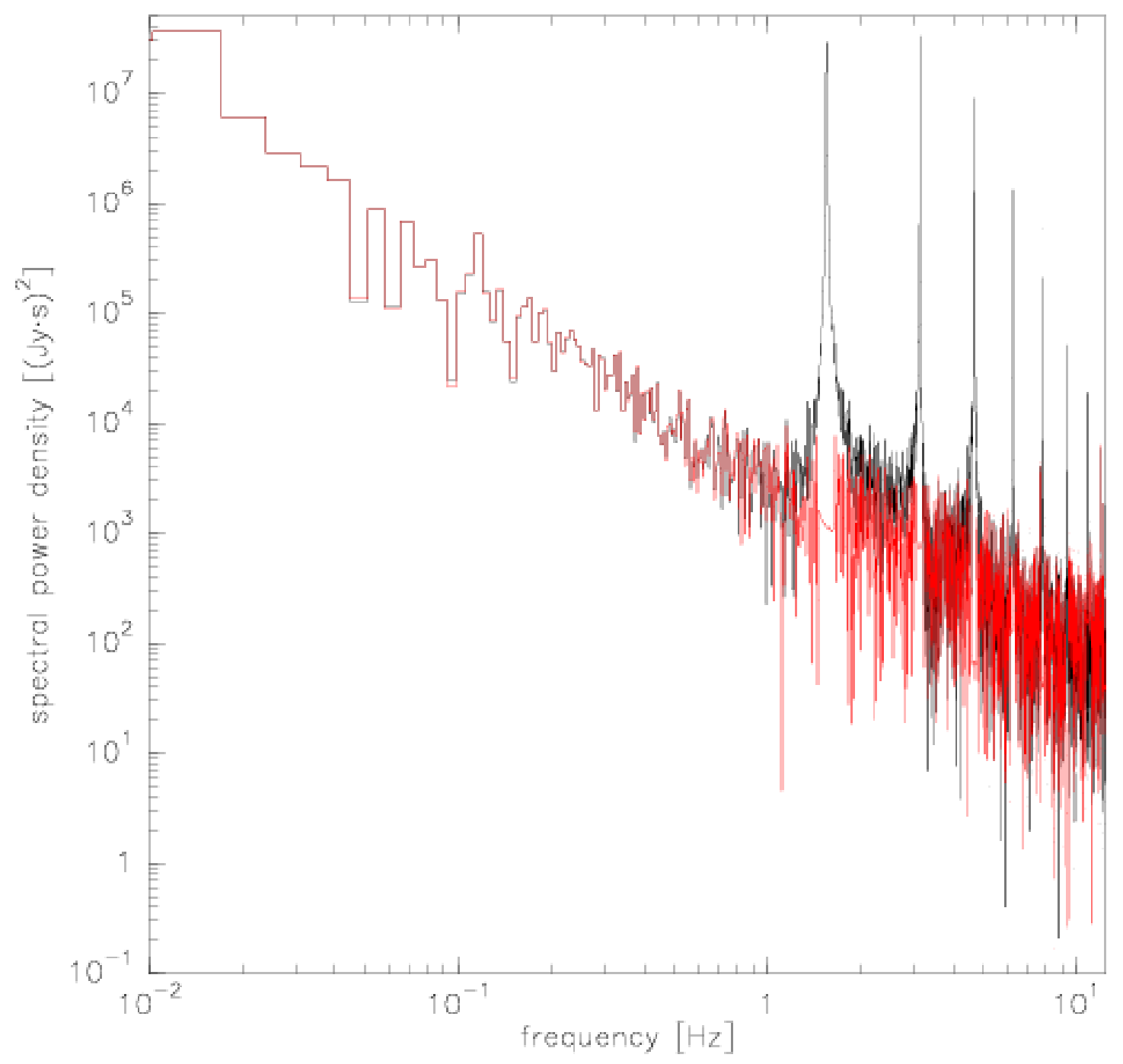

Fig. 5.- Spectral power density of the time series of a single on-the-fly map with a spiral stroke pattern, as received from the central pixel of the bolometer array before (black) and after (red) removal of the beating (the spectrum does not reach a zero power density due to the noise bias). The $1 / \mathrm{f}$ noise from the atmospheric fluctuations is visible at frequencies below $\sim 3 \mathrm{~Hz}$. 


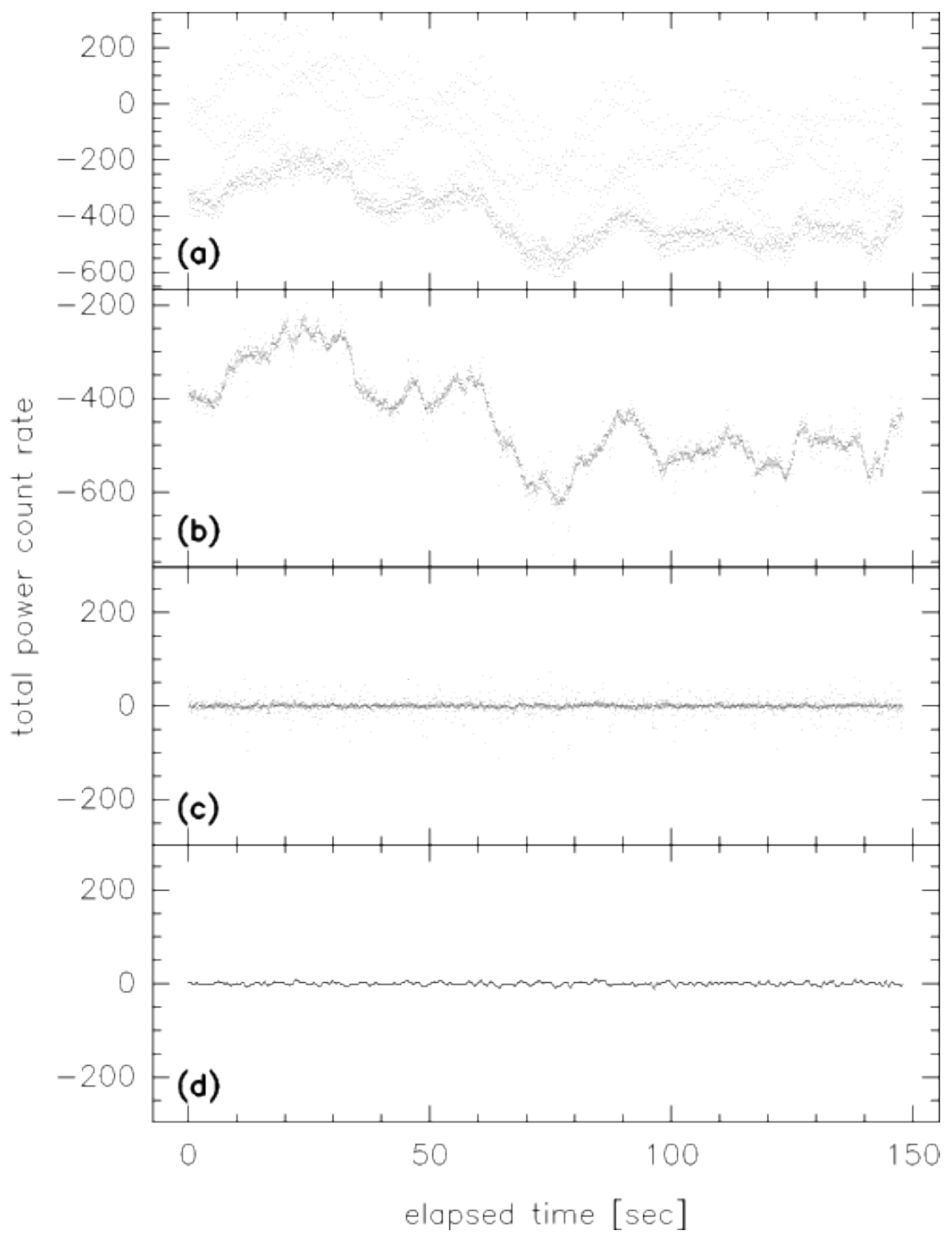

Fig. 6.- Demonstration of the data reduction steps. From top to bottom: (a) Time series of raw data from the central LABOCA pixel, for a single on-the-fly map with spiral stroke pattern. (b) Same after removal of total power beating, (c) after removal of correlated noise, (d) after removal of high-frequency noise (wavelet filter, see appendix D). The vertical scale in (b)-(d) is fixed so as to show the noise suppression. 


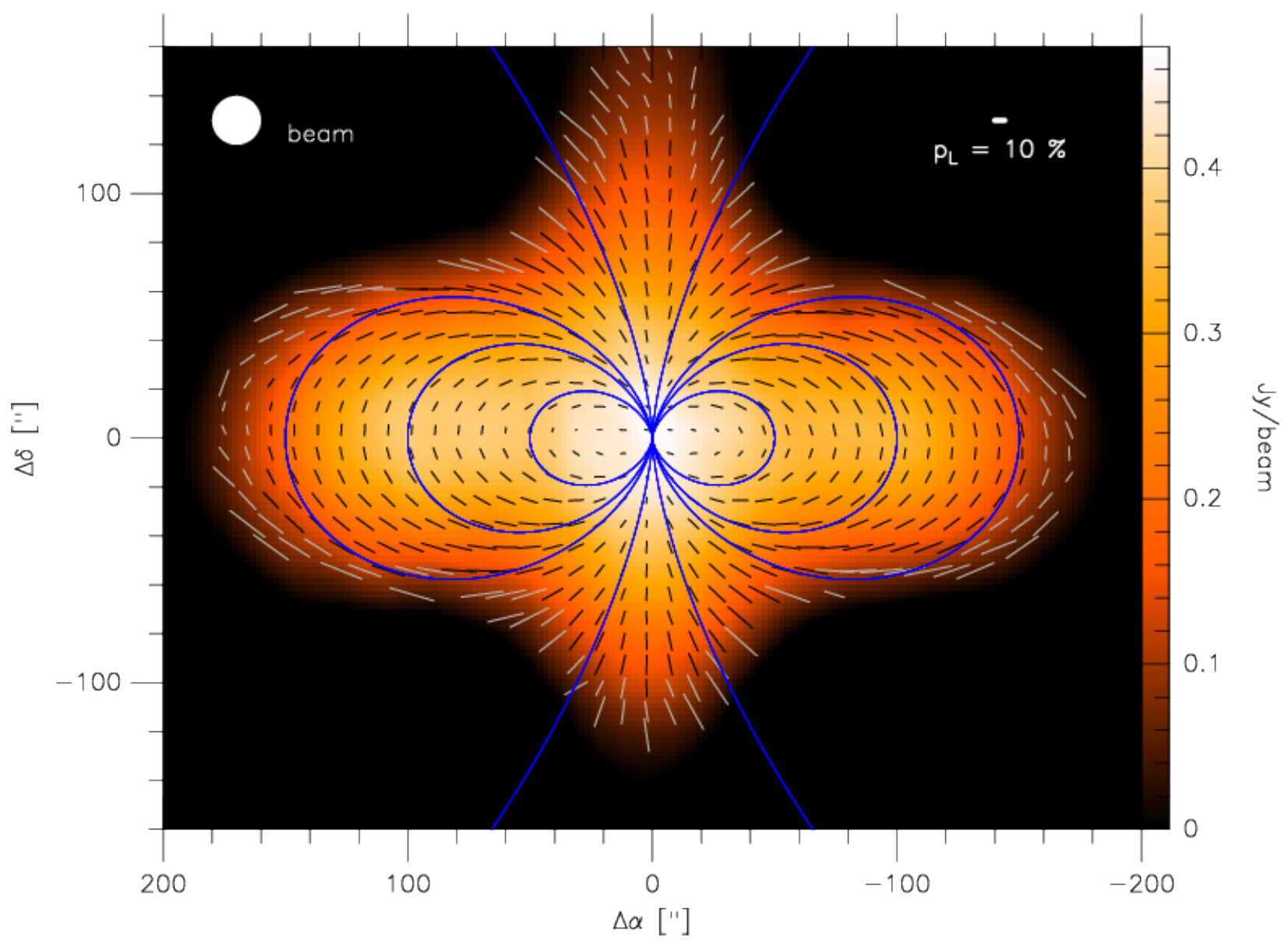

Fig. 7.- Reconstruction of a test source with linear polarization along dipole field lines (blue contours, $p_{\mathrm{L}}=10 \%$ ). The linear polarization deduced by the data reduction is shown as black or white vectors. The emission in Stokes $I$ (color scale) consists of three Gaussian brightness distributions. The simulation includes atmospheric total power fluctuations and a total power beating. The on-the-fly sampling of this test source is the same as for Fig. 9. Polarization vectors are shown for a Stokes $I$ emission above $1 \mathrm{mJy}$. For details see section 3.2. The beam size (20" FWHM) and a $10 \%$ linear polarization are shown in the upper left and right corners, respectively. 


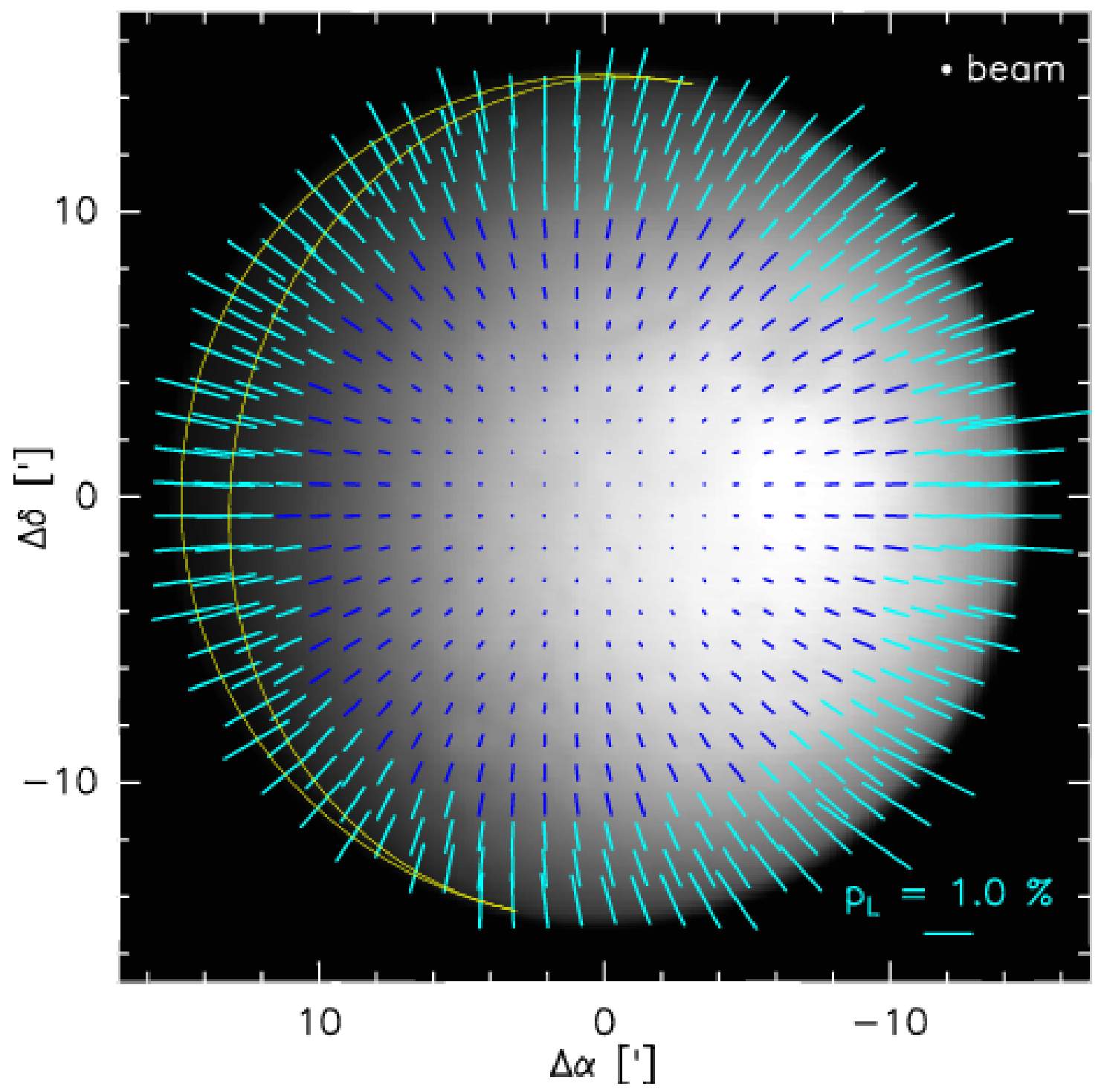

Fig. 8.- Linear polarization of the lunar submillimeter emission. A fractional linear polarization of $1 \%$ is indicated in the lower right corner. The yellow contour shows the position of the terminator. The PolKa beam (20" FWHM) is indicated in the upper right corner. 

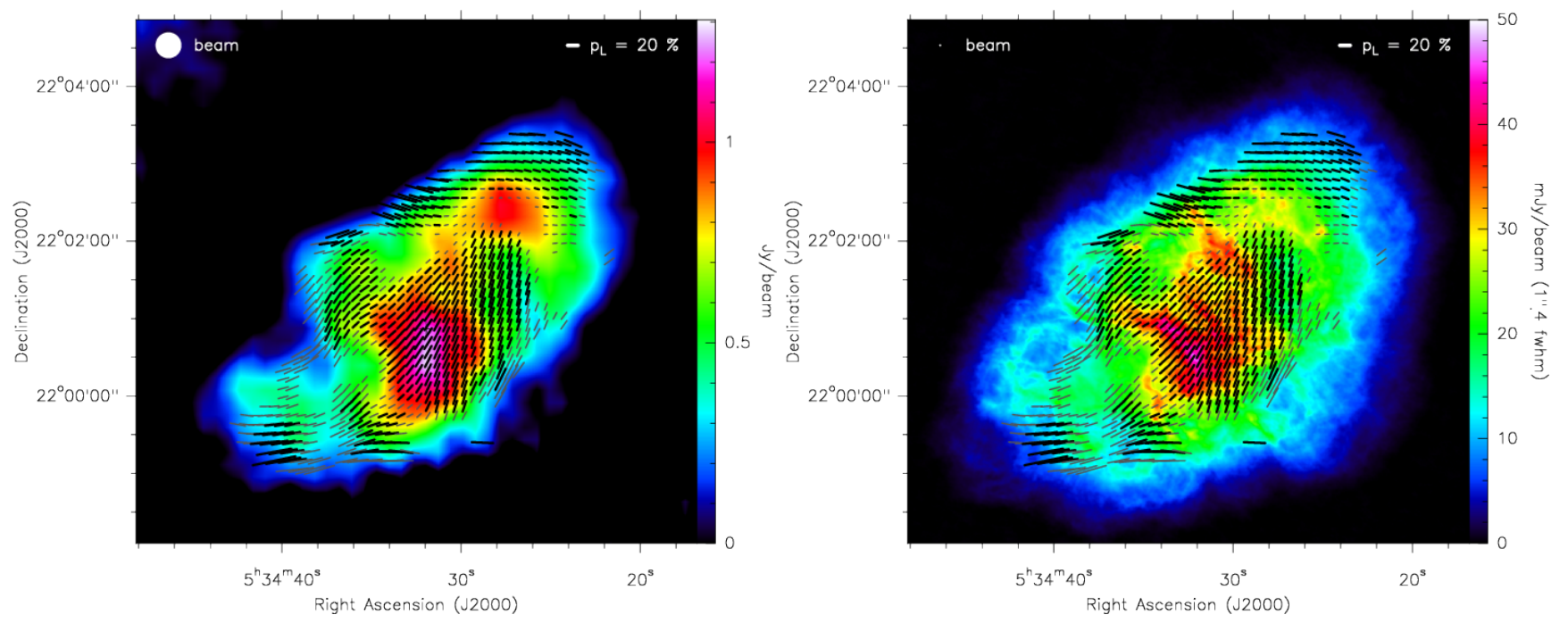

Fig. 9. - $\lambda 870 \mu \mathrm{m}$ polarization vectors (corrected for instrumental polarization) in Tau A. Polarizations above $3 \sigma_{\mathrm{p}_{\mathrm{L}}}$ (i.e., $\sigma_{\psi} \leq 9.5$ ) are shown in black, those with $2 \sigma_{\mathrm{p}_{\mathrm{L}}} \leq p_{\mathrm{L}}<3 \sigma_{\mathrm{p}_{\mathrm{L}}}$ in gray $\left(\sigma_{\psi} \leq 14.3\right)$. A linear polarization of $20 \%$ is indicated in the top right corners. The black cross marks the pulsar position. Left: with Stokes $\lambda 870 \mu \mathrm{m}$ Stokes I emission underneath (plot scale to the right). The 20" (FWHM) beam is shown in the upper left corner. Right: with VLA $5 \mathrm{GHz}$ continuum (1".4 FWHM, archive data, Bietenholz et al. 2001). 

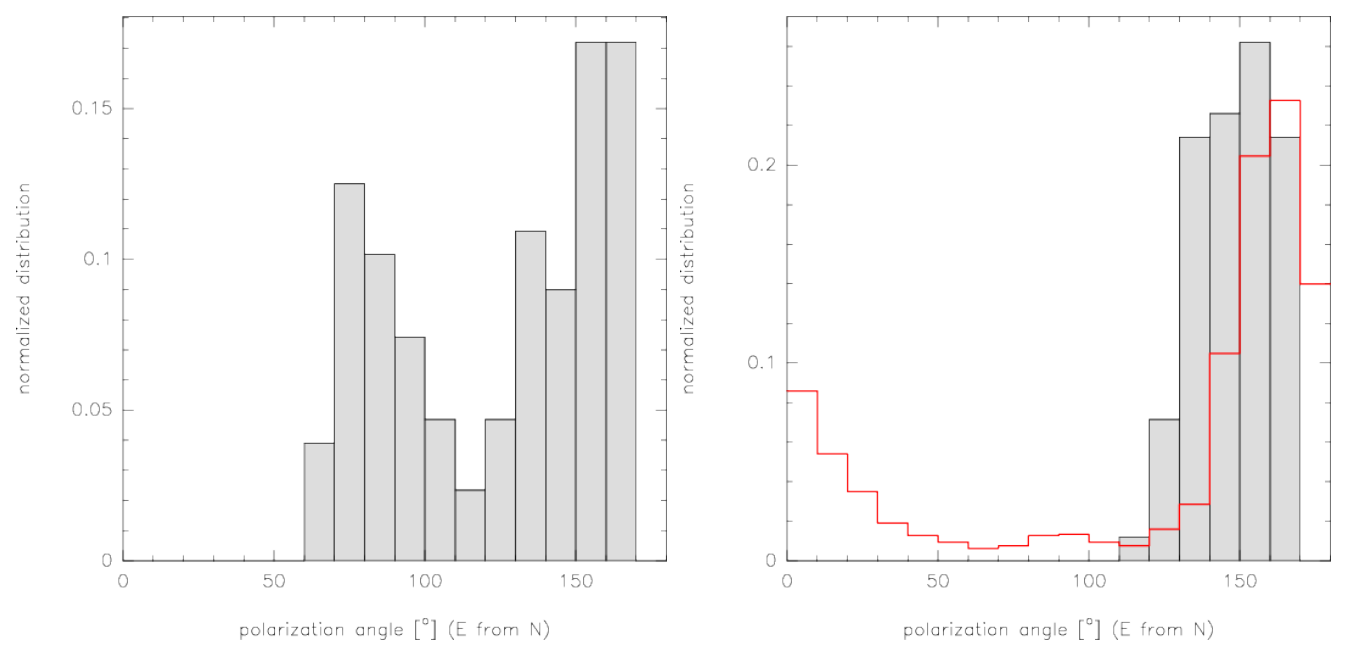

Fig. 10.- Distribution of polarization vectors in Tau A. Left: In the whole synchrotron nebula, above the $0.1 \mathrm{Jy} /$ beam contour. Right: in the inner 100" (filled gray histogram). For comparison, the corresponding distribution for optical data from a central field of the same size (HST/ACS, Moran et al. 2013) is also shown (red histogram). 


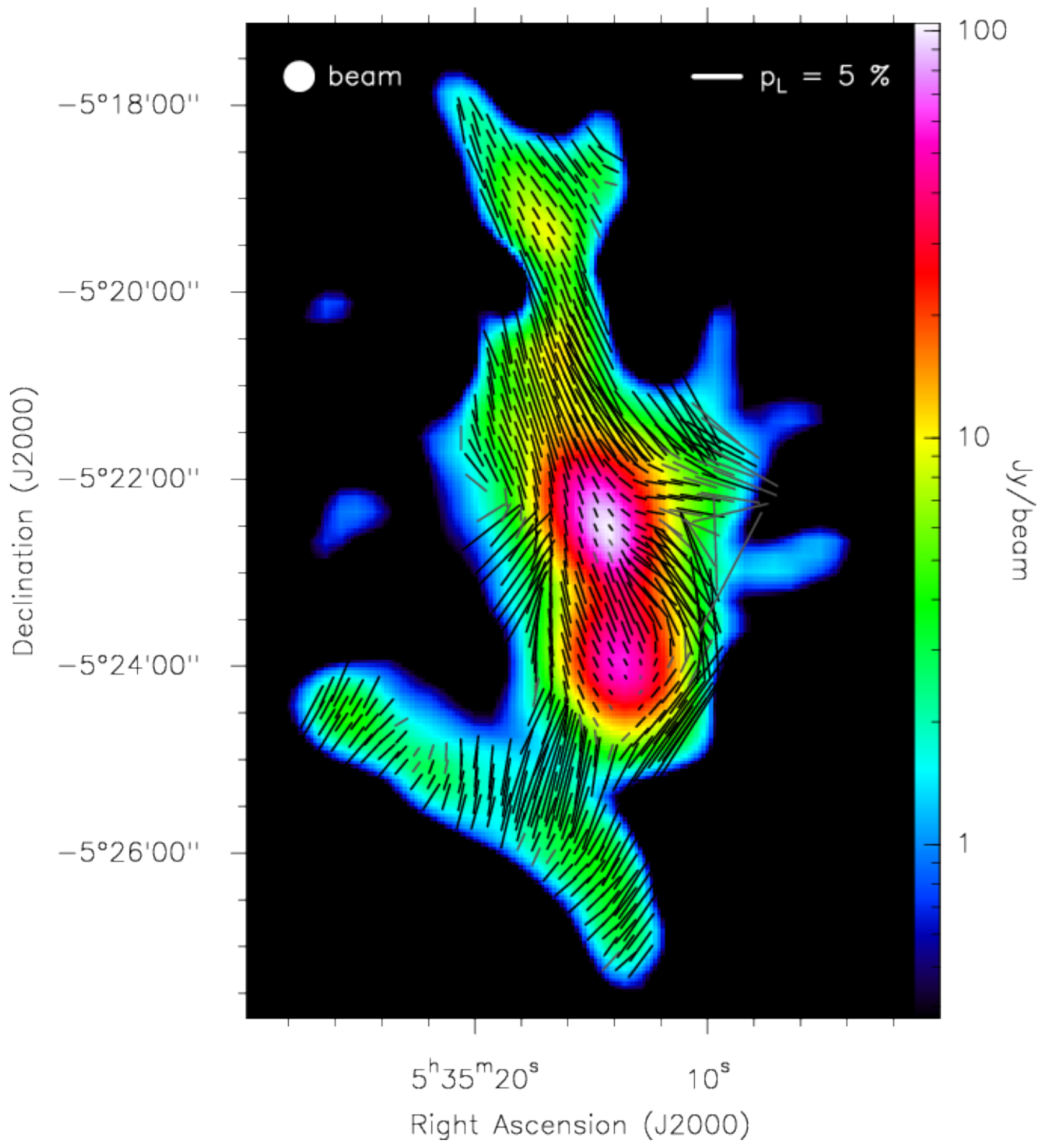

Fig. 11. - $\lambda 870 \mu \mathrm{m}$ polarization vectors, corrected for instrumental polarization, in OMC1 $\left(20^{\prime \prime}\right.$ FWHM, shown in the upper left corner) with Stokes $I$ emission underneath (color plot scale to the right). A linear polarization of $5 \%$ is indicated in the top right corner. Use of black and gray polarization vectors as in Fig. 9 . 

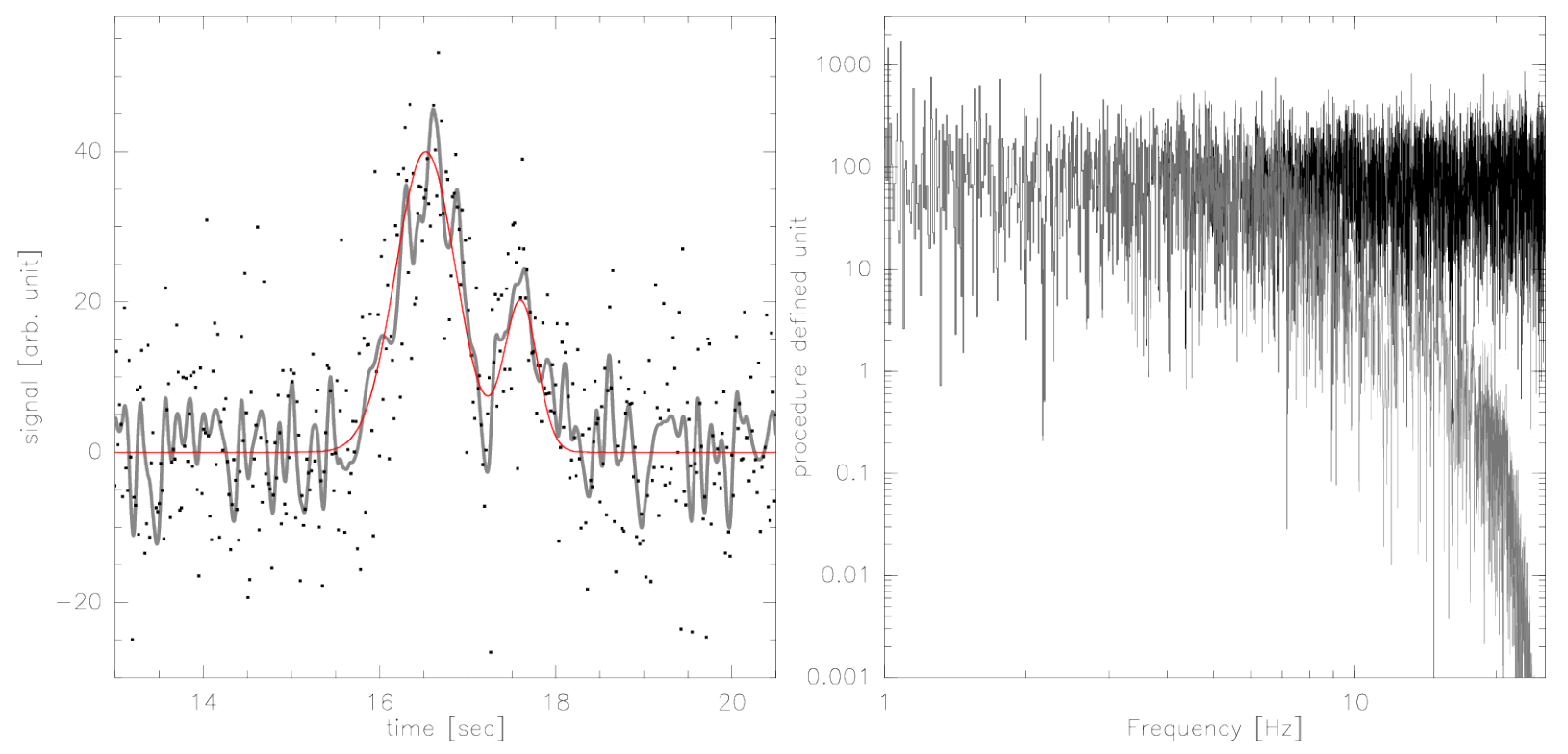

Fig. 12. - Removal of high-frequency noise with a wavelet filter. The test source has a double Gaussian profile and is repeatedly scanned, like in a real on-the-fly map. The signal-to-noise ratio at peak is four. Left: Part of the modeled time series. The red line shows the input model before, the black dots after adding the Gaussian noise. The thick gray line shows the profile after application of the wavelet filter. Right: Spectral power density before (black) and after (gray) application of the wavelet filter. 